\title{
Many-valued hybrid logic
}

Hansen, Jens Ulrik; Bolander, Thomas; Braüner, Torben

Published in:

Journal of Logic and Computation

Link to article, DOI:

10.1093/logcom/exv040

Publication date:

2018

Document Version

Peer reviewed version

Link back to DTU Orbit

Citation (APA):

Hansen, J. U., Bolander, T., \& Braüner, T. (2018). Many-valued hybrid logic. Journal of Logic and Computation, 28(5), 883-908. https://doi.org/10.1093/logcom/exv040

\section{General rights}

Copyright and moral rights for the publications made accessible in the public portal are retained by the authors and/or other copyright owners and it is a condition of accessing publications that users recognise and abide by the legal requirements associated with these rights.

- Users may download and print one copy of any publication from the public portal for the purpose of private study or research.

- You may not further distribute the material or use it for any profit-making activity or commercial gain

- You may freely distribute the URL identifying the publication in the public portal

If you believe that this document breaches copyright please contact us providing details, and we will remove access to the work immediately and investigate your claim. 


\title{
Many-Valued Hybrid Logic
}

\author{
Jens Ulrik Hansen, Thomas Bolander, and Torben Braüner
}

\begin{abstract}
In this paper we define a family of many-valued semantics for hybrid logic, where each semantics is based on a finite Heyting algebra of truth-values. We provide sound and complete tableau systems for these semantics. Moreover, we show how the tableau systems can be made terminating and thereby give rise to decision procedures for the logics in question. Our many-valued hybrid logics turn out to be "intermediate" logics between intuitionistic hybrid logic and classical hybrid logic in a specific sense explained in the paper. Our results show that many-valued hybrid logic is indeed a natural enterprise.
\end{abstract}

Keywords: Modal logic, hybrid logic, many-valued logic, intuitionistic hybrid logic, tableau systems.

\section{Introduction}

Classical hybrid logic is obtained by adding further expressive procedureower to ordinary, classical modal logic in the form of a second sort of propositional symbols called nominals, and moreover, by adding so-called satisfaction operators. Each nominal is assumed to be true at exactly one world (state), and consequently, nominals can be regarded as names of worlds. If $i$ is a nominal and $\varphi$ is an arbitrary formula, then a new formula $@_{i} \varphi$ called a satisfaction statement can be formed ( $@_{i}$ is called a satisfaction operator). The satisfaction statement $@_{i} \varphi$ expresses that the formula $\varphi$ is true at the particular world at which $i$ is true. In this manner, hybrid logic enables references to particular worlds and reasoning about what is true at such worlds.

Hybrid logic offers other advantages over classical modal logic than merely increased expressivity. In contrast to classical modal logic, hybrid logic is proof-theoretically wellbehaved. Natural deduction systems satisfying normalisation, cut-free Gentzen systems, and terminating tableau systems can all be given for a variety of hybrid logics in uniform ways [7]. Moreover, the metalinguistic machinery introduced into proof systems to deal with numerous modal logics can be completely internalised in hybrid logic [7, 6].

Despite the success and extensive studies of hybrid logic, existing hybrid logics are almost exclusively two-valued. There might be several reasons for this. Maybe there is no use for non-classical hybrid logics? This is hard to believe as non-classical modal logics have found numerous applications. Maybe there are inherent technical difficulties in achieving natural non-classical hybrid logics? The latter concern is our point of departure. We will investigate a general class of many-valued hybrid logics and show that it can be realised in a natural way and has an attractive proof-theory in form of a sound, complete and terminating tableau calculus.

Classical hybrid logic can be viewed as a combination of two logics, namely classical, two-valued logic (where the standard propositional connectives are interpreted in terms of the truth-values true and false) and hybrid modal logic (where modal operators, nominals, and satisfaction operators are interpreted in terms of a set of possible worlds equipped with 
an accessibility relation). The present paper concerns hybrid logic where the two-valued logical basis has been replaced by a many-valued one, providing us with a many-valued semantics for hybrid logic. There are numerous available many-valued logical bases to choose from, and we can not consider all. We will make a simplifying assumption that the set of truth-values is finite. Still, we aim at investigating the most general class of manyvalued hybrid logics possible. Therefore, we have chosen to hybridise the very general many-valued semantics for modal logic introduced by Melvin Fitting in [14, 15, 16].

In Fitting's many-valued modal logic, the set of truth-values is a finite Heyting algebra, that is, a finite lattice with meets and joins (used to interpret conjunction and disjunction) and with relative pseudo-complements (used to interpret implication). Since we need both the diamond and the box modality, and since implication plays a key role in defining the semantics of the box modality, Heyting algebras are a natural choice for the set of truth-values. Moreover, the logic of Fitting has the general feature that it allows not only formulas but also accessibility links to be assigned non-classical truth-values, which is contrary to a number of many-valued modal logics, where acessibility links are only assigned classical truth-values.

The motivation originally used by Fitting for his many-valued modal logic was multiexpert systems [15]. These are systems where a group of experts have individual opinions on the truth of modal issues, and where there is a relation of dominance between the agents. The truth-values can then be taken to be subsets of experts and the truth-value of a formula is the subset of experts who accepts the formula as true. This view is further developed and described in [18]. This motivation can naturally be transferred to our case as well, and would grant the experts increased expressive power. In addition to Fitting's multi-expert motivation, there are a number of applications of many-valued modal logics, mostly in cases where fuzziness, uncertainty, or truth-likeness play a role. Many-valued modal logic can, for instance, be used for fuzzy similarity-based reasoning [21] or for reasoning about fuzzy beliefs [26].

Deciding on a many-valued modal logic to hybridise does not completely determine what the many-valued hybrid logic will look like. There are several possible choices for the semantics of both nominals and satisfaction operators. Nonetheless, it turns out that if we want to retain as many of the useful properties of classical hybrid logic as possible, then there is a unique best choice of many-valued semantics for the hybrid machinery. In Section 2, we will present this many-valued semantics as well as discuss the alternative choices and their limitations.

The number of existing papers on non-classical hybrid logic is quite limited. Most of these focus on intuitionistic hybrid logic $[9,7,10,20]$. As we will later see, there is a close relationship between a version of intuitionistic hybrid logic and the many-valued hybrid logics of the present paper. This relationship is fully elaborated in Section 3. Many-valued hybrid logics appear to only previously have been investigated in an earlier version of this paper [25] and by Didier Galmiche and Yakoub Salhi in [19].

In [19], Galmiche and Salhi introduce a sequence of finite-valued hybrid logics $G H_{1}$, $G H_{2}, \ldots$ together with two infinite-valued hybrid logics $G H_{\infty}$ and $W G H_{\infty}$. For any natural number $n$, the logic $G H_{n}$ is an $n+1$ valued logic where the truth-values are rational numbers, including 0 and 1 , taken from the real unit interval $[0,1]$. The set of truthvalues is equipped with the usual order on the rational numbers and the interpretation of the logical connectives is the standard generalisation of the interpretation in classical 
two-valued hybrid logic, which is equivalent to $G H_{1}$. The interpretation of the modal operators involves a many-valued interpretation of the accessibility relation together with the relative pseudo-complement of the implicit Heyting algebra structure on the set of truth-values. It follows that for any natural number $n$, the semantics of $G H_{n}$ is a special case of the many-valued semantics considered in the present paper. ${ }^{1}$ A main contribution of [19] is to prove that the logics $G H_{n}$ as well as the logic $W G H_{\infty}$ are decidable using a proof-search procedure involving a set of sequent rules. Decidability of $G H_{\infty}$ is left open. Note that since the logics $G H_{n}$, where $n$ is any natural number, are many-valued hybrid logics in our sense, decidability of these logics also follows from our decidability result (which was already published in [25]), though our method is quite different from theirs.

Tableau systems for hybrid logic is a well-established area of research within the prooftheory of hybrid logic and includes a long line of work, such as $[2,1,5,4,22,3]$. One of the advantages of hybrid logic tableau systems is that metalinguistic machinery, such as prefixes, can be completely internalised resulting in conceptually simpler systems. Consequently, destructive tableau systems such as Fittings [16] can be avoided and general methods for showing termination of tableau systems can be applied. In Section 4, we provide a tableau system that is sound and complete with respect to the many-valued semantics defined in Section 2. The tableau system is a natural (but non-trivial) generalisation of the existing tableau system for two-valued logic from [5]. In particular, if our tableau system is restricted to a set with only two truth-values, then modulo minor reformulations and the deletion of superfluous rules, the tableau system obtained is identical to the existing tableau system for two-valued hybrid logic. Moreover, to deal with the Heyting algebra of truth-values, considerable inspiration is taken form Fittings tableau system for many-valued modal logic [16], even though our tableau system is non-destructive. Completeness of our tableau system is shown in Section 6 .

As mentioned, in addition to being sound and complete, our tableau system is also terminating, providing us with a decision procedure for the logic. Hence our many-valued hybrid logic is decidable and has the finite model property. Since all formulas of Fitting's many-valued modal logic are also formulas of our many-valued hybrid logic, our tableau calculus automatically provides a terminating, sound and complete calculus for Fitting's logic. From this we can conclude that also Fitting's logic is decidable and has the final model property. Fitting himself provides a sound and complete tableau calculus for his logic in [16], but the question of termination, and thus decidability, is not addressed.

In Section 5, we will present a proof of termination of our tableau calculus inspired by the general procedure used in [4]. Finally, we will end the paper with a conclusion and some remarks on future work.

\section{A Many-Valued Hybrid Logic}

In this section a language for many-valued hybrid logic, denoted MVHL, is defined and a semantics provided. The language includes the global modalities $E$ ("somewhere") and $A$ ("everywhere"). The global modalities will be used to motivate our choice of semantics for nominals. However, they will be excluded from the logic for which we present a sound

\footnotetext{
${ }^{1}$ The logic $G H_{\infty}$ is obtained from $G H_{n}$ by replacing the $n+1$ truth-values by the whole real unit interval $[0,1]$. The logic $W G H_{\infty}$ is obtained from $G H_{\infty}$ by restricting to models where all infima and suprema needed in the interpretation of the modal operators are respectively minima and maxima (the letter 'W' stands for 'Witnessed').
} 
and complete tableau calculus.

A finite Heyting algebra $\mathcal{H}$ is a finite lattice with meet $\square$ and join $\sqcup$, such that for all $a$ and $b$ in $\mathcal{H}$ there is a greatest element $x$ of $\mathcal{H}$ satisfying $a \sqcap x \leq b$. The element $x$ is called the relative pseudo-complement of $a$ with respect to $b$ and is denoted by $a \Rightarrow b$. The largest and smallest elements of $\mathcal{H}$ are denoted $T$ and $\perp$, respectively. We will be using finite Heyting algebras as the space of truth-values for our many-valued logic. ${ }^{2}$

\subsection{Syntax for MVHL}

Let a countable infinite set of propositional variables PROP and a countable infinite set of nominals NOM be given.

DEFINITION 1 (MVHL-formulas). The set of MVHL-formulas over a finite Heyting algebra $\mathcal{H}$ is given by:

$$
\varphi::=p|a| i|(\varphi \wedge \varphi)|(\varphi \vee \varphi)|(\varphi \rightarrow \varphi)| \square \varphi|\diamond \varphi| @_{i} \varphi|E \varphi| A \varphi,
$$

where $p \in \mathrm{PROP}, a \in \mathcal{H}$, and $i \in \mathrm{NOM}$.

In general, we will use $i, j, k, \ldots$ for nominals and $a, b, c, \ldots$ for elements of $\mathcal{H}$.

\subsection{Semantics for MVHL}

MVHL-formulas over $\mathcal{H}$ are interpreted in Kripke models in which the accessibility links are allowed to take values in $\mathcal{H}$. This is inspired by [16]. A model $\mathcal{M}$ over $\mathcal{H}$ is a tuple $\mathcal{M}=\langle W, R, \mathbf{n}, \nu\rangle$, where $W$ is the set of worlds, and $R$ a mapping $R: W \times W \rightarrow \mathcal{H}$ called the accessibility relation (by slight abuse of language, since it is not formally a relation). $\mathbf{n}$ is a function interpreting the nominals, i.e., $\mathbf{n}:$ NOM $\rightarrow W$. Finally the valuation $\nu: W \times$ PROP $\rightarrow \mathcal{H}$ assigns truth-values to the propositional variables at each world.

Given a model $\mathcal{M}=\langle W, R, \mathbf{n}, \nu\rangle$, we inductively extend the valuation $\nu$ to all formulas in the way shown in Figure 1, where $w \in W$. An MVHL-formula $\varphi$ is said to be valid (relative to $\mathcal{H}$ ) if $\varphi$ is a formula over $\mathcal{H}$, and if $\nu(w, \varphi)=\top$ for all models $\mathcal{M}=\langle W, R, \mathbf{n}, \nu\rangle$ over $\mathcal{H}$ and all $w \in W .^{3}$

The semantics chosen for the hybrid logical constructions is discussed in the following. As in classical two-valued hybrid logic, nominals denote single worlds specified by $\mathbf{n}$. Furthermore, we have chosen to assign each nominal $i$ the value $T$ in the world it denotes $(\mathbf{n}(i))$, and $\perp$ in all other worlds. This way nominals still denote single worlds. If we had only required nominals to be $T$ in exactly one world and different from $T$ in all others, nominals would still denote single worlds. However, then other problems would arise, which will be discussed in the next subsection. The semantics for $@_{i} \varphi$ is obvious, its truth-value is simply the truth-value of $\varphi$ at the world $i$ denotes. This is motived by the semantics of $@_{i} \varphi$ in standard hybrid logic. The semantics chosen for the global

\footnotetext{
${ }^{2}$ In order to give reasonable semantics for $\wedge$ and $\vee$, the set of truth-values $\mathcal{H}$ must be a lattice. A complete lattice would be sufficient if the accessibility links were two-valued, but since we wish these to take values in $\mathcal{H}$, it has to be a Heyting algebra. For further discussions on the choice of a finite Heyting algebra as the set of truth-values, see [15, 16].

${ }^{3}$ Note that by this definition we do not define a single logic (i.e. a set of valid formulas), but a family of logics, namely one for each finite Heyting algebra $\mathcal{H}$. However, we will sometime refer to MVHL as a logic instead of a family of logics, in which case we think of the logic resulting from keeping a finite Heyting algebra fixed. In most cases the fixed Heyting algebra will be arbitrary and thus, what we claim will be true for all logics in the family MVHL.
} 


$$
\begin{aligned}
\nu(w, a) & :=a \text { for } a \in \mathcal{H} \\
\nu(w, i) & :=\left\{\begin{array}{c}
\top \quad \text { if } \mathbf{n}(i)=w \\
\perp \text { otherwise }
\end{array}\right. \\
\nu(w, \varphi \wedge \psi) & :=\nu(w, \varphi) \sqcap \nu(w, \psi) \\
\nu(w, \varphi \vee \psi) & :=\nu(w, \varphi) \sqcup \nu(w, \psi) \\
\nu(w, \varphi \rightarrow \psi) & :=\nu(w, \varphi) \Rightarrow \nu(w, \psi) \\
\nu(w, \square \varphi) & :=\prod\{R(w, v) \Rightarrow \nu(v, \varphi) \mid v \in W\} \\
\nu(w, \diamond \varphi) & :=\bigsqcup\{R(w, v) \sqcap \nu(v, \varphi) \mid v \in W\} \\
\nu\left(w, @_{i} \varphi\right) & :=\nu(\mathbf{n}(i), \varphi) \\
\nu(w, A \varphi) & :=\prod\{\nu(v, \varphi) \mid v \in W\} \\
\nu(w, E \varphi) & :=\bigsqcup\{\nu(v, \varphi) \mid v \in W\}
\end{aligned}
$$

Figure 1. Valuation of MVHL-formulas in a Kripke model.

modalities $A$ and $E$ reflect the fact that these modalities are simply the global versions of the modalities $\square$ and $\diamond$.

Our choice of semantics for the nominals and the satisfaction operators allows us to preserve the well-known logical equivalence $@_{i} \varphi \leftrightarrow E(i \wedge \varphi)$ and $@_{i} \varphi \leftrightarrow A(i \rightarrow \varphi)$ from classical (two-valued) hybrid logic, since the following hold in MVHL: ${ }^{4}$

$$
\begin{aligned}
& \nu\left(w, @_{i} \varphi\right)=\nu(\mathbf{n}(i), \varphi)=\bigsqcup\{\nu(v, i) \sqcap \nu(v, \varphi) \mid v \in W\}=\nu(w, E(i \wedge \varphi)) \\
& \nu\left(w, @_{i} \varphi\right)=\nu(\mathbf{n}(i), \varphi)=\prod\{\nu(v, i) \Rightarrow \nu(v, \varphi) \mid v \in W\}=\nu(w, A(i \rightarrow \varphi)) .
\end{aligned}
$$

Another property of our semantics is the following:

$$
\nu\left(w, @_{i} \diamond j\right)=\nu(\mathbf{n}(i), \diamond j)=\bigsqcup\{R(\mathbf{n}(i), v) \sqcap \nu(v, j) \mid v \in W\}=R(\mathbf{n}(i), \mathbf{n}(j)) .
$$

This identity expresses that reachability of the world denoted by $j$ from the world denoted by $i$ is correctly captured by the formula $@_{i} \diamond j$. This also holds in classical (two-valued) hybrid logic. Identity between worlds denoted by nominals can also be expressed as in two-valued hybrid logic, since the following holds in MVHL:

$$
\nu\left(w, @_{i} j\right)=\top \text { iff } \mathbf{n}(i)=\mathbf{n}(j) .
$$

Finally, an additional nice property of the choice of semantics is that the logic collapses to classical two-valued hybrid logic when $\mathcal{H}=\{\top, \perp\}$.

\subsection{Alternative semantics for MVHL}

Alternative semantics for hybrid logic are possible in the many-valued setting. We will not consider alternative semantics for the basic modal logical connectives, since we decided to

\footnotetext{
${ }^{4}$ Using the fact that in a Heyting algebra: $\top \sqcap a=a, \perp \sqcap a=\perp, a \sqcup \perp=a, \top \Rightarrow a=a$ and $\perp \Rightarrow a=\top$.
} 


\begin{tabular}{|c||c|c|c|c|c|c|}
\hline & $\mathrm{MVHL}$ & $\mathrm{MVHL}_{1}$ & $\mathrm{MVHL}_{2}$ & $\mathrm{MVHL}_{3}$ & $\mathrm{MVHL}_{4}$ & $\mathrm{MVHL}_{5}$ \\
\hline \hline$\nu\left(w, @_{i} j\right)=\top$ iff $\bar{i}=\bar{j} ?$ & yes & yes & no & yes & $\begin{array}{c}\text { no } \\
(\Rightarrow \text { holds })\end{array}$ & $\begin{array}{c}\text { no } \\
(\text { undef. })\end{array}$ \\
\hline$\nu\left(w, @_{i} \diamond j\right)=R(\bar{i}, \bar{j}) ?$ & yes & $\begin{array}{c}\text { no } \\
(\geq \text { holds })\end{array}$ & $\begin{array}{c}\text { no } \\
(\geq \text { holds })\end{array}$ & $\begin{array}{c}\text { no } \\
(\geq \text { holds })\end{array}$ & no & $\begin{array}{c}\text { no } \\
(\text { undef. })\end{array}$ \\
\hline$\nu(w, A(i \rightarrow \varphi))=\nu\left(w, @_{i} \varphi\right) ?$ & yes & $\begin{array}{c}\text { no } \\
(\leq \text { holds })\end{array}$ & $\begin{array}{c}\text { no } \\
(\leq \text { holds })\end{array}$ & $\begin{array}{c}\text { no } \\
(\leq \text { holds })\end{array}$ & $\begin{array}{c}\text { yes } \\
(\text { by def. })\end{array}$ & no \\
\hline$\nu\left(w, @_{i} \varphi\right)=\nu(w, E(i \wedge \varphi)) ?$ & yes & $\begin{array}{c}\text { no } \\
(\leq \text { holds })\end{array}$ & $\begin{array}{c}\text { yes } \\
(\text { by def. })\end{array}$ & $\begin{array}{c}\text { yes } \\
(\text { by def. })\end{array}$ & $\begin{array}{c}\text { no } \\
(\leq \text { holds })\end{array}$ & yes \\
\hline $\begin{array}{c}\text { Collapses to classical hybrid } \\
\text { logic when } \mathcal{H}=\{\top, \perp\} ?\end{array}$ & yes & yes & yes & yes & yes & yes \\
\hline
\end{tabular}

Figure 2. Comparison between the logics $\mathrm{MVHL}, \mathrm{MVHL}_{1}, \ldots, \mathrm{MVHL}_{5}$

construct our logic as an extension of the many-valued logic introduced in [14]. We will not consider alternative semantics for the global modalities either, as these are defined as the global versions of the modalities $\square$ and $\diamond$, as usual. Consequently, we will only consider alternative semantics for the nominals and the satisfaction operator. We will consider five alternative versions of the semantics, resulting in five different logics, named $\mathrm{MVHL}_{1^{-}}$ $\mathrm{MVHL}_{5}$. The logics will be compared to MVHL based on the four properties mentioned in the previous subsection, namely whether: 1) the formula $@_{i} j$ expresses equality between the worlds denoted by $i$ and $j ; 2)$ the formula $@_{i} \diamond j$ expresses the value of the accessibility relation between the worlds denoted by $i$ and $j ; 3)$ the formulas $@_{i} \varphi, E(i \wedge \varphi)$ and $A(i \rightarrow \varphi)$ are logically equivalent; 4) the logic collapses to classical hybrid logic when $\mathcal{H}=\{\top, \perp\}$. Our findings are summarised in Figure 2 and show that MVHL is the only one among the six logics that satisfy all the four properties of classical hybrid logic. ${ }^{5}$ The detailed proofs of why the properties hold for some of the logics and fail for others, are omitted. The detailed proofs can be found in [24, Ch. 3].

\section{$M V H L_{1}$}

The first obvious alternative definition of the semantics would be to allow a nominal $i$ to take other values than $\perp$ in the worlds that $i$ does not denote. Formally, we replace $\mathbf{n}$ by a mapping $\mathbf{n}: W \times \mathrm{NOM} \rightarrow \mathcal{H}$, where $\mathbf{n}$ is required to satisfy that for all $i \in \mathrm{NOM}$ there is a unique world $w \in W$ such that $\nu(w, i)=\top$ (this unique world will be denoted by $\bar{i}$ ).

\footnotetext{
${ }^{5}$ Note that we only explored the five alternative logics we considered most obvious, but there might still be other alternative ways of defining the semantics of nominals and satisfaction operators in a manyvalued settings, which retain the mentioned nice properties of classical hybrid logic. Contrarily, one could argue that since we are searching for a many-valued hybrid logic it is not necessarily desirable to preserve as many properties of classical hybrid logic as possible - we should be searching for a truly fuzzy version of hybrid logic. This may be so, in which case the logic $\mathrm{MVHL}_{5}$ might be the best choice. However, as suggested in the introduction, we have chosen to view two-valued hybrid logic as a combination of two-valued logic and hybrid logic, and many-valued hybrid logic as a combination of many-valued logic and hybrid logic. From this perspective, it makes sense to seek to preserve as many as possible of the core hybrid-logical properties.
} 
The semantics of the nominals and satisfaction operators are then replaced by:

$$
\begin{aligned}
\nu(w, i) & :=\mathbf{n}(w, i) \\
\nu\left(w, @_{i} \varphi\right) & :=\nu(\bar{i}, \varphi)
\end{aligned}
$$

The logic that results from this alternative semantics will be called $\mathrm{MVHL}_{1}$. Note that it collapses to classical hybrid logic when $\mathcal{H}=\{\top, \perp\}$. Furthermore, because nominals receive the value $T$ in exactly one world, identity between worlds can still be expressed. More precisely:

$$
\nu\left(w, @_{i} j\right)=\top \operatorname{iff} \bar{i}=\bar{j} .
$$

However, the logic $\mathrm{MVHL}_{1}$ is different from MVHL. Accessibility between worlds are no longer expressible in the usual way, since $\nu\left(w, @_{i} \diamond j\right)=R(\bar{i}, \bar{j})$ no longer holds in general. The inequality $\nu\left(w, @_{i} \diamond j\right) \leq R(\bar{i}, \bar{j})$ does hold, though. With respect to the global modalities we have the following inequalities:

$$
\nu(w, A(i \rightarrow \varphi)) \leq \nu\left(w, @_{i} \varphi\right) \leq \nu(w, E(i \wedge \varphi)) .
$$

However, in $\mathrm{MVHL}_{1}$ these inequalities may be strict. ${ }^{6}$ Thus, the satisfaction operator cannot be defined by the global modalities in the usual way.

\section{$M V H L_{2}$}

If we want the formula $@_{i} \varphi$ to be definable by the global modality $E$ in the usual way,we could change (4) to

$$
\nu\left(w, @_{i} \varphi\right):=\bigsqcup\{\nu(v, i) \sqcap \nu(v, \varphi) \mid v \in W\},
$$

and obtain another alternative semantics. The logic resulting from this choice of semantics will be called $\mathrm{MVHL}_{2}$. Note that the truth-values that the nominal $i$ takes in other worlds than $\bar{i}$ now matters for the truth-value of $@_{i} \varphi$. Furthermore, $@_{i} \varphi$ and $E(i \wedge \varphi)$ become equivalent by definition, but the inequality $\left.\nu(w, A(i \rightarrow \varphi)) \leq \nu\left(w, @_{i} \varphi\right)\right)$ may still be strict in some cases.

The logic $\mathrm{MVHL}_{2}$ still collapses to classical hybrid logic when $\mathcal{H}=\{\top, \perp\}$. However, in $\mathrm{MVHL}_{2}, \nu\left(w, @_{i} j\right)=\top$ no longer guarantees that $\bar{i}=\bar{j}$ (but the opposite holds). Hence, identity between worlds are no longer expressible in the usual way. As with $\mathrm{MVHL}_{1}$, the inequality $R(\bar{i}, \bar{j}) \leq \nu(w, @ i \diamond j)$ may be strict and thus accessibility between worlds are also not expressible in the standard way either.

\section{$\mathrm{MVHL}_{3}$}

Note that $\mathrm{MVHL}_{2}$ (as well as $\mathrm{MVHL}$ and $\mathrm{MVHL}_{1}$ ) is not a logic as such, but a family of logics, namely one logic for each choice of $\mathcal{H}$. It turns out that there is a subfamily of $\mathrm{MVHL}_{2}$ where identity between worlds can be expressed by $@_{i} j$. This family, which will be denoted $\mathrm{MVHL}_{3}$, is obtained by only allowing certain kinds of Heyting algebras $\mathcal{H}$, namely those where $T$ is join-irreducible. An element $a \neq \perp$ of a Heyting algebra $\mathcal{H}$ is

\footnotetext{
${ }^{6}$ The fact that the formulas $A(i \rightarrow \varphi)$ and $E(i \wedge \varphi)$ are not equivalent might seems strange, however, if one considers a hybrid logic where nominals are true in at most one world and not exactly one world, the formulas are not equivalent either [23].
} 
called join-irreducible if for all $x, y \in \mathcal{H}, a=x \sqcup y$ implies that either $a=x$ or $a=y$. It can then be showed that for all finite Heyting algebras $\mathcal{H}, \top$ is join-irreducible (in $\mathcal{H}$ ) if and only the following property holds:

(*) For all models $\mathcal{M}=\langle W, R, \nu, \mathbf{n}\rangle$, for all $w \in W$, and for all nominals $i$ and $j$ (with the semantics of $\left.\mathrm{MVHL}_{2}\right): \nu\left(w, @_{i} j\right)=\top$ iff $\bar{i}=\bar{j}$.

Thus, due to the requirement on $\mathcal{H}$, equality between worlds is expressible in $\mathrm{MVHL}_{3}$ by formulas of the form $@_{i} j$. The formula $@_{i} \diamond j$ also obtain the nice property that $\nu\left(w, @_{i} \diamond j\right)=\top$ if and only if $R(\bar{i}, \bar{j})=\top$. Again the inequality $R(\bar{i}, \bar{j}) \leq \nu\left(w, @_{i} \diamond j\right)$ holds in general. Nevertheless, it may be a strict inequality if $R(\bar{i}, \bar{j})<T$.

Note that the class of Heyting algebras where $T$ is join-irreducible is not a small and insignificant class. For instance, whenever $\mathcal{H}$ is a linear order, $T$ becomes irreducible. Hence, the sets of truth-values for all the logics $G H_{n}$ of [19], mentioned in the introduction, are Heyting algebras where $T$ is joint-irreducible.

\section{$\mathrm{MVHL}_{4}$}

Instead of defining the semantics of $@_{i}$ from the formula $E(i \wedge \varphi)$ as in $\mathrm{MVHL}_{2}$, one could define it from the formula $A(i \rightarrow \varphi)$. In other worlds, we could replace (4) in $\mathrm{MVHL}_{2}$ by

$$
\nu\left(w, @_{i} \varphi\right):=\prod\{\nu(v, i) \Rightarrow \nu(v, \varphi) \mid v \in W\} .
$$

The resulting (family of) logic(s) will be denoted $\mathrm{MVHL}_{4}$. Now, $\nu\left(w, @_{i} j\right)=T$ does imply that $\bar{i}=\bar{j}$, however, $\bar{i}=\bar{j}$ does not imply that $\nu\left(w, @_{i} j\right)=T$. In fact, in this logic the formulas $@_{i} j$ and $@_{j} i$ are no longer equivalent. The formula $@_{i} j$ now expresses the property that

$$
\nu(w, i) \leq \nu(w, j), \quad \text { for all } w \in W .
$$

Furthermore, in this logic the equalities $\nu\left(w, @_{i} \diamond j\right)=R(\bar{i}, \bar{j})$ and $\nu(w, A(i \rightarrow \varphi))=$ $\nu(w, E(i \wedge \varphi))$ do not hold in general. However, as for all the previously discussed logics, it collapses to classical hybrid logic when $\mathcal{H}=\{\top, \perp\}$.

\section{$M V H L_{5}$}

So far we have only considered logics in which nominals denote single worlds, by only being $T$ in exactly one world. An alternative option is to let nominals point out sets of worlds whose truth-values assigned to the nominals "sums up to T". ${ }^{7}$ With this semantics for nominals, definition (4) is not well-defined (since $\bar{i}$ is not well-defined). However, (5) can still be used, resulting in the logic we call $\mathrm{MVHL}_{5}$. We will not delve into the details of this logic (see [24] for details), just mention that it is a drastic change from classical hybrid logic since nominals now point out sets of worlds. However, when $\mathcal{H}=\{\top, \perp\}$ the logic still collapses to classical hybrid logic.

\footnotetext{
${ }^{7}$ Formally, we assume that for each model and nominal $i$ there is a unique finite set $W_{i} \subseteq W$ such that $\cup_{w \in W_{i}} \ltimes(w, i)=\top$ and there are no proper subset of $W_{i}$ with this property. Again, the details are given in $[24$, Ch. 3].
} 


\section{The relation to intuitionistic hybrid logic}

As pointed out in [15], there is a close relationship between the many-valued modal logic presented there and intuitionistic modal logic. We will now consider the relation between our many-valued hybrid logic and the intuitionistic hybrid logic from $[9,7]$ (which in turn is a hybridisation of an intuitionistic modal logic introduced in a tense-logical version in [12]). In the following, we will only consider those MVHL-formulas that belong to intuitionistic hybrid logic, that is, the only atomic formulas we consider are ordinary propositional symbols, nominals, and the symbol $\perp$ (no truth-values except $\perp$ are allowed). We first define an appropriate notion of an intuitionistic model. Occasionally such models will be called Kripke models to avoid confusion with other sorts of models for intuitionistic modal and hybrid logic.

DEFINITION 2. A restricted model for intuitionistic hybrid logic is a tuple

$$
\left(W, \leq, D,\left\{R_{w}\right\}_{w \in W},\left\{\nu_{w}\right\}_{w \in W}\right)
$$

where

1. $W$ is a non-empty finite set partially ordered by $\leq$;

2. $D$ is a non-empty set;

3. for each $w, R_{w}$ is a binary relation on $D$ such that $w \leq v$ implies $R_{w} \subseteq R_{v}$; and

4. for each $w, \nu_{w}$ is a function that to each ordinary propositional symbol $p$ assigns a subset of $D$ such that $w \leq v$ implies $\nu_{w}(p) \subseteq \nu_{v}(p)$.

This notion of model can be seen as a restricted variant of the notion of a model of $[9,7] .^{8}$ Note that the set $D$ is to be understood as the set of possible worlds and is used to interpret the modal and hybrid parts of the language (occasionally together with the set $W$ ). The elements of the set $W$ are states of knowledge and for any such state $w$, the relation $R_{w}$ is the set of known relationships between possible worlds and the set $\nu_{w}(p)$ is the set of possible worlds at which $p$ is known to be true. Note that the definition requires that the epistemic partial order $\leq$ preserves these kinds of knowledge, that is, if an advance to a greater state of knowledge is made, then what is known is preserved.

Given a restricted model $\mathfrak{M}=\left(W, \leq, D,\left\{R_{w}\right\}_{w \in W},\left\{\nu_{w}\right\}_{w \in W}\right)$, an assignment is a function $\mathbf{n}$ that to each nominal assigns an element of $D$. The semantic relation $\mathfrak{M}, \mathbf{n}, w, d=\varphi$ is defined by induction, where $w$ is an element of $W, \mathbf{n}$ is an assignment, $d$ is an element

\footnotetext{
${ }^{8}$ Compare to Definition 8.1, p. 172, of [7]. The differences are the following: i) In [7], the set $W$ need not be finite. ii) Instead of $D$ there is a family $\left\{D_{w}\right\}_{w \in W}$ of non-empty sets such that $w \leq v$ implies $D_{w} \subseteq D_{v}, R_{w}$ is a binary relation on $D_{w}$, and $\nu_{w}(p)$ is a subset of $D_{w}$. iii) There is a family $\left\{\sim_{w}\right\}_{w \in W}$ where $\sim_{w}$ is an equivalence relation on $D_{w}$ such that $w \leq v$ implies $\sim_{w} \subseteq \sim_{v}$ and such that if $d \sim_{w} d^{\prime}, e \sim_{w} e^{\prime}$, and $d R_{w} e$, then $d^{\prime} R_{w} e^{\prime}$, and similarly, if $d \sim_{w} d^{\prime}$ and $d \in \nu_{w}(p)$, then $d^{\prime} \in \nu_{w}(p)$. The equivalence relations are used for the interpretation of nominals. Such a model for intuitionistic hybrid logic corresponds to a standard model for intuitionistic first-order logic with equality, where equality is interpreted using the equivalence relations, cf. [28].
} 
of $D$, and $\varphi$ is a formula.

$$
\begin{array}{rll}
\mathfrak{M}, \mathbf{n}, w, d=p & \text { iff } & d \in \nu_{w}(p) \\
\mathfrak{M}, \mathbf{n}, w, d \models i & \text { iff } & d=\mathbf{n}(i) \\
\mathfrak{M}, \mathbf{n}, w, d \models \varphi \wedge \psi & \text { iff } & \mathfrak{M}, \mathbf{n}, w, d \models \varphi \text { and } \mathfrak{M}, \mathbf{n}, w, d=\psi \\
\mathfrak{M}, \mathbf{n}, w, d \models \varphi \vee \psi & \text { iff } & \mathfrak{M}, \mathbf{n}, w, d \models \varphi \text { or } \mathfrak{M}, \mathbf{n}, w, d \models \psi \\
\mathfrak{M}, \mathbf{n}, w, d \models \varphi \rightarrow \psi & \text { iff } & \text { for all } v \geq w, \\
& & \mathfrak{M}, \mathbf{n}, v, d \models \varphi \text { implies } \mathfrak{M}, \mathbf{n}, v, d \models \psi \\
\mathfrak{M}, \mathbf{n}, w, d \models \perp & \text { iff } & \text { falsum } \\
\mathfrak{M}, \mathbf{n}, w, d \models \square \varphi & \text { iff } & \text { for all } v \geq w, \text { for all } e \in D, \\
& & d R_{v} e \text { implies } \mathfrak{M}, \mathbf{n}, v, e \models \varphi \\
\mathfrak{M}, \mathbf{n}, w, d=\diamond \varphi & \text { iff } & \text { for some } e \in D, d R_{w} e \text { and } \mathfrak{M}, \mathbf{n}, w, e=\varphi \\
\mathfrak{M}, \mathbf{n}, w, d \models \varliminf_{i} \varphi & \text { iff } & \mathfrak{M}, \mathbf{n}, w, \mathbf{n}(i) \models \varphi \\
\mathfrak{M}, \mathbf{n}, w, d \models A \varphi & \text { iff } & \text { for all } v \geq w, \text { for all } e \in D, \mathfrak{M}, \mathbf{n}, v, e=\varphi \\
\mathfrak{M}, \mathbf{n}, w, d \models E \varphi & \text { iff } & \text { for some } e \in D, \mathfrak{M}, \mathbf{n}, w, e \models \varphi
\end{array}
$$

A formula $\varphi$ is said to be restricted intuitionistically valid if $\mathfrak{M}, \mathbf{n}, w, d=\varphi$ holds for all choices of $\mathfrak{M}, \mathbf{n}, w$ and $d$.

This semantics can be looked upon in two different ways: As indicated above, it can be seen as a restricted variant of the semantics of $[9,7]$, but it can also be seen as a hybridised version of a semantics from [15]. In the latter paper, the epistemic worlds are thought of as experts and the epistemic partial order is thought of as a relation of dominance between experts: One expert dominates another one if whatever the first expert says is true is also said to be true by the second expert.

As pointed out in [15], the restricted intuitionistic semantics for modal logic is in a certain sense equivalent to the many-valued semantics. This also holds in the hybrid-logical case. To be precise: Given a restricted model $\mathfrak{M}=\left(W, \leq, D,\left\{R_{w}\right\}_{w \in W},\left\{\nu_{w}\right\}_{w \in W}\right)$, cf. Definition 2, and an assignment $\mathbf{n}$, it can be shown that the $\leq$-closed subsets of $W$ ordered by $\subseteq$ constitute a finite Heyting algebra $\mathcal{H}$. Moreover, a many-valued model $\left(D, R^{*}, \mathbf{n}, \nu^{*}\right)$ over $\mathcal{H}$ can be defined by letting

- $R^{*}(d, e)=\left\{w \in W \mid d R_{w} e\right\}$ and

- $\nu^{*}(d, p)=\left\{w \in W \mid d \in \nu_{w}(p)\right\}$.

Given this machinery, the following theorem can be proved.

THEOREM 3. For any formula $\varphi$, it holds that $\nu^{*}(d, \varphi)=\{w \in W \mid \mathfrak{M}, \mathbf{n}, w, d=\varphi\}$.

Proof. A straightforward extension of the corresponding proof in [15].

Thus, the restricted intuitionistic semantics can be simulated by the many-valued semantics. The above implies that if a formula is valid independently of the particular Heyting algebra chosen, then it is restricted intuitionistically valid.

There is also a correspondence in the opposite direction, enabling the many-valued semantics to be simulated by the restricted intuitionistic semantics. Given a finite Heyting algebra $\mathcal{H}$ and a many-valued model $(D, R, \mathbf{n}, \nu)$ over $\mathcal{H}$, a restricted model $\mathfrak{M}=$ $\left(W, \subseteq, D,\left\{R_{w}^{*}\right\}_{w \in W},\left\{\nu_{w}^{*}\right\}_{w \in W}\right)$ can be defined by letting

- $W=\{w \mid w$ is a proper prime filter in $\mathcal{H}\}$, 
- $d R_{w}^{*} e$ if and only if $R(d, e) \in w$, and

- $d \in \nu_{w}^{*}(p)$ if and only if $\nu(d, p) \in w$.

Details can be found in [15]. Given this, the following theorem can be proved.

THEOREM 4. For any formula $\varphi$, it holds that $\mathfrak{M}, \mathbf{n}, w, d=\varphi$ if and only if $\nu(d, \varphi) \in w$.

Proof. A straightforward extension of the proof in [15].

This theorem implies that if a formula is restricted intuitionistically valid, then it is also valid in the many-valued semantics relative to any given finite Heyting algebra. This follows from the observation that the set $\{\top\}$ is a proper prime filter in any Heyting algebra.

Thus, in the above sense the restricted intuitionistic semantics for hybrid logic is equivalent to the many-valued semantics. It is an interesting question whether there is such an equivalence if instead of the restricted models one considers the more general models of $[9,7] .{ }^{9}$ This question is left for future work.

Now, let us say that a hybrid-logical formula is intuitionistically valid if it is valid with respect to the more general model notion of $[7$, p. 174].

THEOREM 5. Intuitionistic validity implies many-valued validity, and many-valued validity implies classical (two-valued) validity.

Proof. A restricted model can trivially can be considered a general model, so intuitionistic validity implies restricted intuitionistic validity, and in the remark following THEOREM 4 we concluded that restricted intuitionistic validity implies many-valued validity. Moreover, many-valued validity implies classical validity, which follows from the easily proven fact that for any finite Heyting algebra $\mathcal{H}$, if a formula is valid in the many-valued semantics relative to $\mathcal{H}$, then it is also valid in the many-valued semantics relative to $\{\top, \perp\}$.

So many-valued hybrid logics are logics between intuitionistic hybrid logic and classical hybrid logic. A three-valued logic from [15] provides an example of a many-valued logic strictly between intuitionistic and classical hybrid logic. In this logic, there is a truthvalue $m$ distinct from $\perp$ and $T$ such that $\perp \leq m \leq T$. A classically valid formula which is not valid in the three-valued logic is the formula $\neg \diamond \neg \varphi \rightarrow \square \varphi$, where negation $\neg \psi$ is an abbreviation of $\psi \rightarrow \perp$. A formula valid in the three-valued logic, but not intuitionistically valid, is the formula $\square \neg \neg \varphi \rightarrow \neg \neg \square \varphi$. This is a well-known example of a modal-logical formula which is not intuitionistically valid, but which is only falsifiable by a Kripke model having an infinite set of epistemic worlds. ${ }^{10}$ The formula is valid in the three-valued semantics since the restricted intuitionistic semantics simulating the threevalued semantics has a finite set of epistemic worlds (there are two proper prime filters in the Heyting algebra $\{\perp, m, \top\}$ of truth-values).

Many-valued hybrid logics being "intermediate" logics between intuitionistic hybrid logic and classical hybrid logic is similar to the fuzzy hybrid logics of [19] mentioned

\footnotetext{
${ }^{9} \mathrm{As}$ indicated in the previous footnote, in the intuitionistic semantics of $[9,7]$, nominals are interpreted using a family $\left\{\sim_{w}\right\}_{w \in W}$ of equivalence relations, not identity. This seems to imply that in an equivalent many-valued semantics, nominals should be allowed to take on arbitrary truth-values, not just $T$ and $\perp$.

${ }^{10}$ The formula demonstrates that intuitionistic modal logic does not have the finite model property with respect to Kripke models. The origin of the counterexample is [27].
} 
earlier. The logics of [19] actually constitute a linearly ordered set of logics between intuitionistic hybrid logic and classical hybrid logic, that is, the sets of valid formulas in the respective logics can be ordered as $G H_{1} \supset G H_{2} \supset \ldots \supset W G H_{\infty} \supset G H_{\infty}$. The logic $G H_{1}$ is equivalent to classical two-valued hybrid logic, and the valid formulas of $G H_{\infty}$ includes intuitionistically valid formulas.

\section{A tableau calculus for MVHL}

In the following we will present a tableau calculus for MVHL. The calculus is parametrised by $\mathcal{H}$ in the sense that for each choice of a finite Heyting algebra $\mathcal{H}$, there is a corresponding set of tableau rules for the MVHL-formulas over $\mathcal{H}$. The basic notions for tableaus are defined as usual (see, e.g., [13]). The formulas occurring in our tableaus will all be of the form $@_{i}(a \rightarrow \varphi)$ or $@_{i}(\varphi \rightarrow a)$ prefixed either a $T$ or an $F$, where $i \in$ NOM and $a \in \mathcal{H}$. That is, the formulas occurring in our tableaus will be signed formulas of hybrid logic. A signed formula of the form $T @_{i}(a \rightarrow \varphi)$ is used to express that the formula $a \rightarrow \varphi$ is true at $i$, that is, receives the value $\top$ at $i$. If $\nu(\mathbf{n}(i), a \rightarrow \varphi)=\top$ then, by definition of $\nu, a \Rightarrow \nu(\mathbf{n}(i), \varphi)=\top$. By definition of relative pseudo-complement, we then get that $\top$ is the greatest element of $\mathcal{H}$ satisfying $a \wedge T \leq \nu(\mathbf{n}(i), \varphi)$. In other words, we simply have $a \leq \nu(\mathbf{n}(i), \varphi)$. Thus, a formula $T @_{i}(a \rightarrow \varphi)$ expressed that the truth-value of $\varphi$ at $i$ is greater than or equal to $a$. Symmetrically, a signed formula of the form $T @_{i}(\varphi \rightarrow a)$ expresses that the truth-value of $\varphi$ at $i$ is less than or equal to $a$. Dually, a signed formula of the form $F @_{i}(a \rightarrow \varphi)\left(F @_{i}(\varphi \rightarrow a)\right)$ expresses that the truth-value of $\varphi$ at $i$ is not greater than or equal to (less than or equal to) $a$.

The tableau rules are divided into four classes: Branch Closing Rules, Non-modal Rules, Modal Rules and Hybrid Rules. The Branch Closing Rules and Non-modal Rules are direct translations of Fitting's corresponding rules for the pure modal case [16].

\section{Branch Closing Rules}

A tableau branch $\Theta$ is said to be closed if one of the following holds:

1. $T @_{i}(a \rightarrow b) \in \Theta$, for some $a, b$ with $a \not \leq b$.

2. $F @_{i}(a \rightarrow b) \in \Theta$, for some $a, b$ with $a \leq b, a \neq \perp$, and $b \neq T$.

3. $F @_{i}(\perp \rightarrow \varphi) \in \Theta$, for some formula $\varphi$.

4. $F @_{i}(\varphi \rightarrow \top) \in \Theta$, for some formula $\varphi$.

5. $T @_{i}(b \rightarrow \varphi), F @_{i}(a \rightarrow \varphi) \in \Theta$, for some $a, b$ with $a \leq b$.

6. $T @_{j}(a \rightarrow i), F @_{i}(b \rightarrow j) \in \Theta$, for some $a, b \neq \perp$.

7. $T @_{i}(i \rightarrow a) \in \Theta$, for some nominal $i$ and truth-value $a$ with $a \neq \top$.

The last two conditions, 6 and 7, have no counterpart in Fitting's system, but are required in ours to deal with the semantics chosen for nominals. Note that if a formula $F @_{i}(a \rightarrow i)$ occurs on a branch then the branch can also be closed: In case $a=\perp$, condition 3 immediately implies closure. If $a \neq \perp$, then using the reversal rule $(F \geq)$ (see below), we can add a formula $T @_{i}(i \rightarrow b)$ to the branch, where $b$ is one of the maximal members of $\mathcal{H}$ not above $a$ (where $b$ not above $a$ means $a \not \leq b$ ). Because $b$ is not above $a$, $b$ cannot be $T$. Thus condition 7 implies closure. 


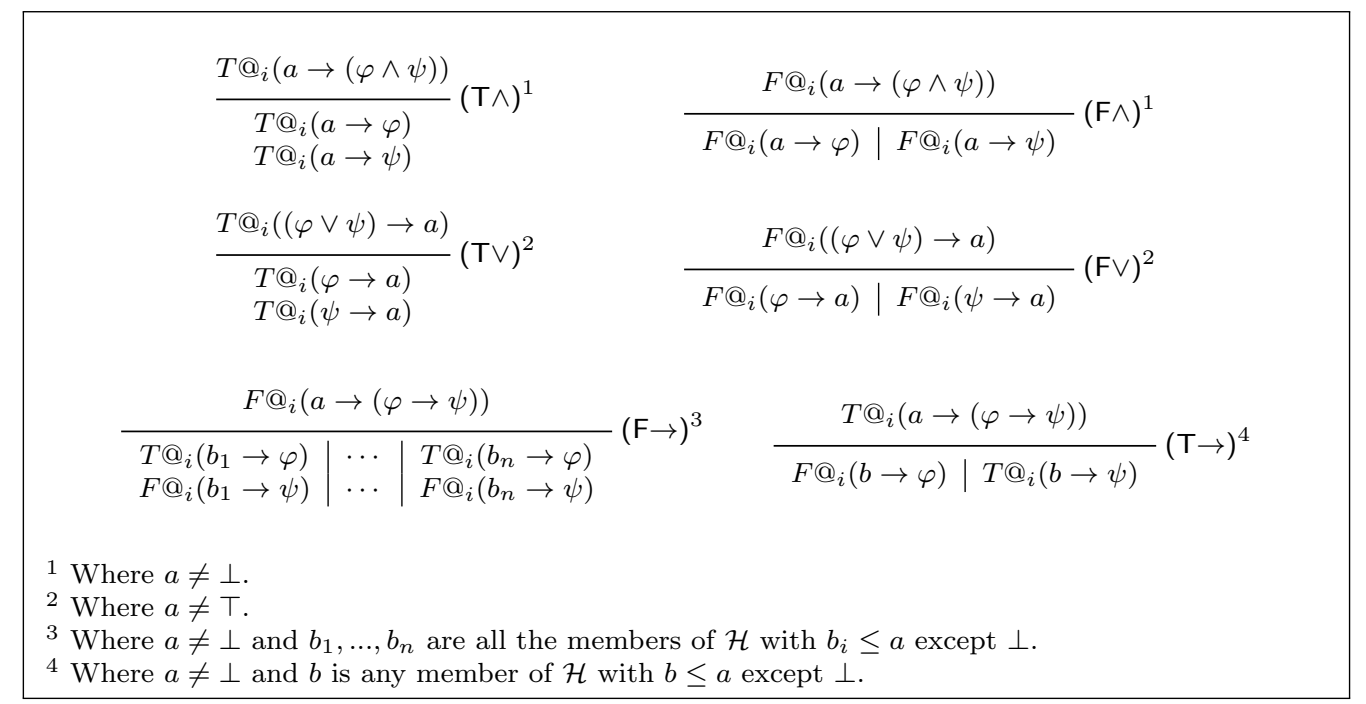

Figure 3. Propositional Rules for MVHL.

\section{Non-modal Rules}

The tableau rules for the propositional connectives and the rules capturing the properties of the Heyting algebra are given in Figure 3 and Figure 4, respectively. The rules of Figure 4 are called reversal rules, as in [16].

\section{Modal Rules}

Our modal rules in Figure 5 differ from the ones of Fitting by heavily employing the hybrid logical machinery. ${ }^{11}$ Some of the tableau rules contain formulas of the form $T @_{i}(a \leftrightarrow \diamond j)$.

\footnotetext{
${ }^{11}$ In [16], the modal tableau rules are so-called destructive rules (see [17] for details) which replaces an entire branch of a tableau with a new branch. The modal rules given here are standard rules that simply add new formulas to the end of existing branches (after possibly splitting these branch first). The rules
}

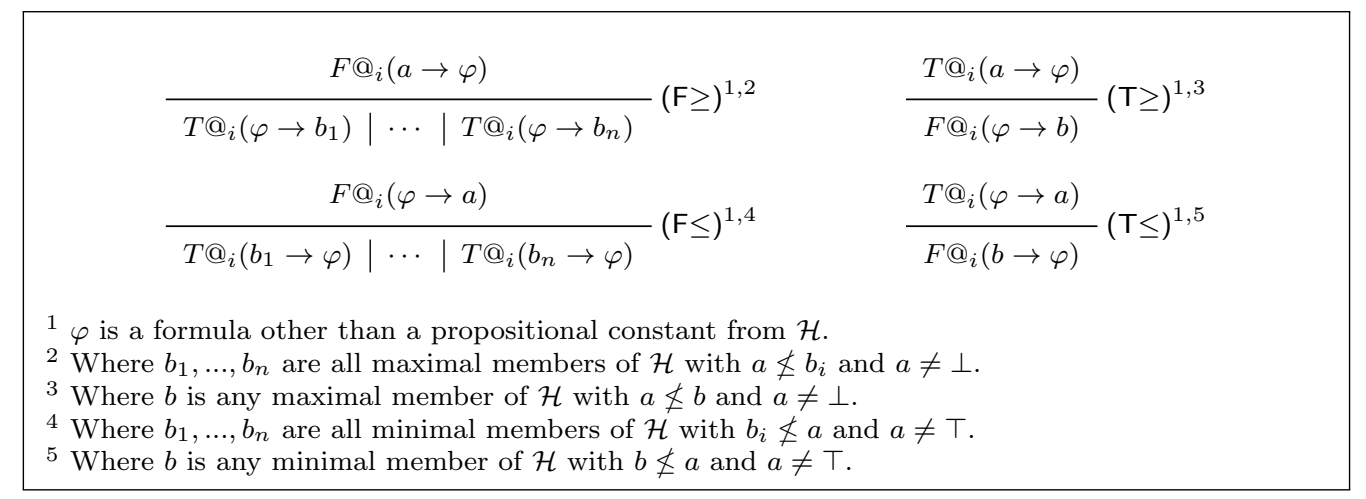

Figure 4. Reversal Rules for MVHL. 


$$
\begin{aligned}
& \begin{array}{c|c|c}
F @_{i}(a \rightarrow \square \varphi) \\
\hline T @_{i}\left(b_{1} \leftrightarrow \diamond j\right) & \cdots & T @_{i}\left(b_{n} \leftrightarrow \diamond j\right) \\
F @_{j}\left(\left(a \sqcap b_{1}\right) \rightarrow \varphi\right) & \cdots & F @_{j}\left(\left(a \sqcap b_{n}\right) \rightarrow \varphi\right)
\end{array} \\
& \frac{T @_{i}(a \rightarrow \square \varphi) \quad T @_{i}(b \rightarrow \diamond j)}{T @_{j}((a \sqcap b) \rightarrow \varphi)}(\mathrm{T} \square)
\end{aligned}
$$

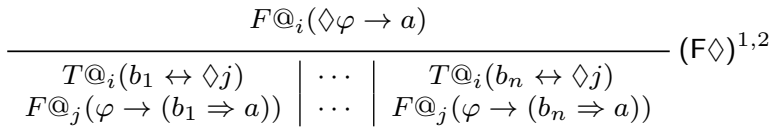

$$
\begin{aligned}
& \frac{T @_{i}(\diamond \varphi \rightarrow a) \quad T @_{i}(b \rightarrow \diamond j)}{T @_{j}(\varphi \rightarrow(b \Rightarrow a))}(\mathrm{T} \diamond)^{2} \\
& \frac{F @_{i}(E \varphi \rightarrow a)}{F @_{j}(\varphi \rightarrow a)}(\mathrm{FE})^{3} \quad \frac{T @_{i}(E \varphi \rightarrow a)}{T @_{j}(\varphi \rightarrow a)}(\mathrm{TE})^{4} \\
& \frac{T @_{i}(a \rightarrow A \varphi)}{T @_{j}(a \rightarrow \varphi)}(\mathrm{TA})^{4} \quad \frac{F @_{i}(a \rightarrow A \varphi)}{F @_{j}(a \rightarrow \varphi)}(\mathrm{FA})^{3}
\end{aligned}
$$

${ }^{1}$ Where $b_{1}, \ldots, b_{n}$ are all the members of $\mathcal{H}$ except $\perp$, and $j$ is a nominal new to the branch.

2 Where the principal premise is a quasi-subformula of the root formula.

3 Where $j$ is a nominal new to the branch.

4 Where $j$ is a nominal already occurring on the branch.

Figure 5. Modal Rules for MVHL. 


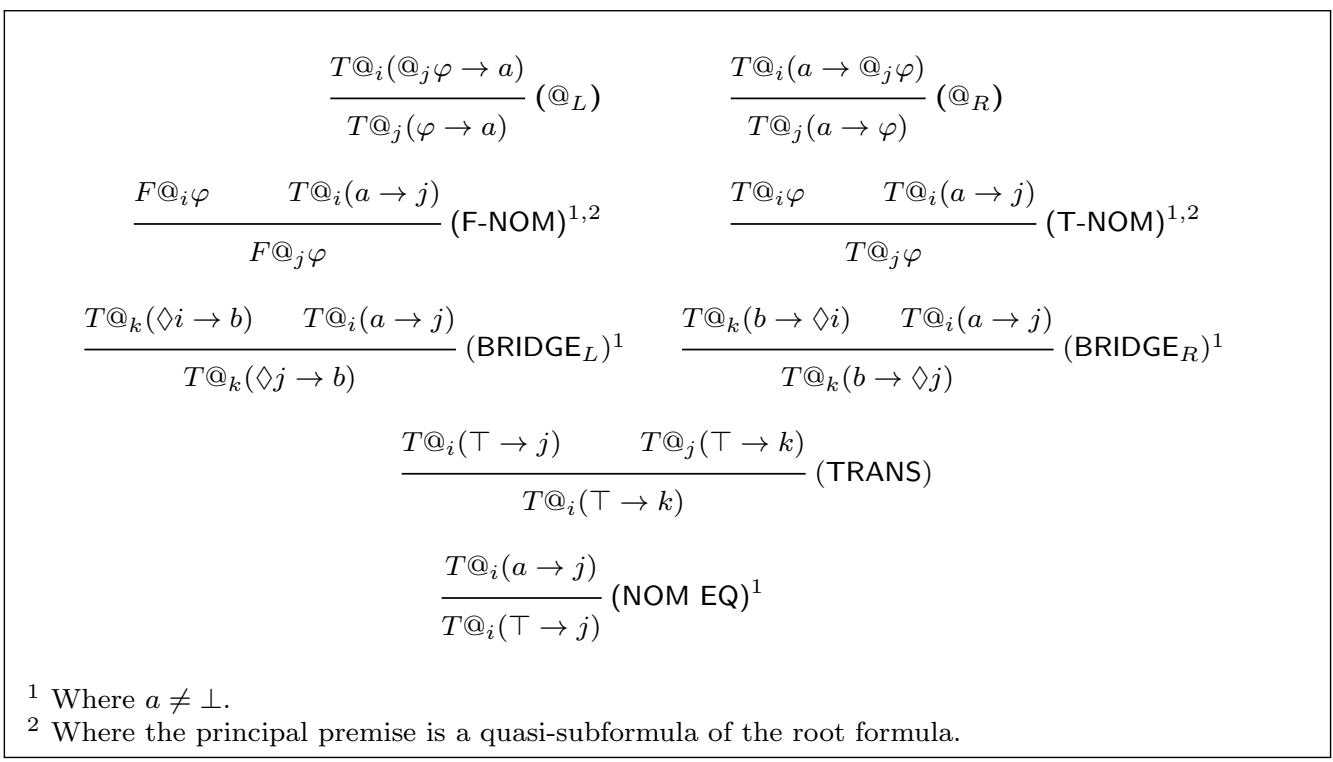

Figure 6. Hybrid Rules for MVHL.

Such formulas are simply used as a shorthand for the occurrence of both $T @_{i}(a \rightarrow \diamond j)$ and $T @_{i}(\diamond j \rightarrow a)$. In each of the rules, the leftmost premise is called the principal premise. In a signed formula of the form $T @_{i}(a \rightarrow \varphi), T @_{i}(\varphi \rightarrow a), F @_{i}(a \rightarrow \varphi)$ or $F @_{i}(\varphi \rightarrow a)$, we call $\varphi$ the body and $i$ theprefix. If $\alpha$ and $\beta$ are two signed formulas such that the body of $\alpha$ is a subformula of the body of $\beta$, then $\alpha$ is said to be a quasi-subformula of $\beta$.

\section{Hybrid Rules}

The hybrid rules of Figure 6 are inspired by the standard rules from classical hybrid logic $[2,5,4]$. Note that two versions of the standard (NOM) rule are needed: (F-NOM) and (TNOM). A further "NOM-like" rule, (NOM EQ), is needed due to the many-valued setting. It ensures that nominals are $T$ in exactly one world.

A tableau proof of a formula $\varphi$ is a closed tableau with root $F @_{i}(\top \rightarrow \varphi)$, where $i$ is an arbitrary nominal not occurring in $\varphi$. The intuition is that the formula $F @_{i}(T \rightarrow \varphi)$ asserts that $\varphi$ does not have the value $T$, and if the tableau closes, this assertion is refuted. If $i$ is a nominal occurring in the root formula of a tableau then $i$ is called a root nominal of the tableau. Other nominals occurring on the tableau are called non-root nominals.

\section{Termination}

The tableau calculus presented above is non-terminating. This is due to the rules for the global modalities. An example of a non-terminating tableau is given in Figure 7 . It is a tableau in the calculus determined by the Heyting algebra $\{\top, \perp\}$. Since the root formula is $F @_{i}(T \rightarrow E \square p)$, it represents an attempt to give a tableau proof of the invalid formula $E \square p$.

of Figure 5 are hence not easily comparable to the rules of [16]. 


$$
\begin{aligned}
& F @_{i}(\top \rightarrow E \square p) \text { root } \\
& T @_{i}(E \square p \rightarrow \perp) \quad(\mathrm{F} \geq) \text { on } 1 \\
& T @_{i}(\square p \rightarrow \perp) \quad(\mathrm{TE}) \text { on } 2 \\
& 4 \quad F @_{i}(T \rightarrow \square p) \quad(\mathrm{T} \leq) \text { on } 3 \\
& X[j / i, k / j]\left\{\begin{array}{lll}
5 & T @_{i}(T \leftrightarrow \Delta j) & (\mathrm{F} \square) \text { on } 4 \\
6 & F @_{j}(T \rightarrow p) & (\mathrm{F} \square) \text { on } 4 \\
7 & T @_{j}(\square p \rightarrow \perp) & (\mathrm{TE}) \text { on } 2 \\
8 & F @_{j}(T \rightarrow \square p) & (\mathrm{T} \leq) \text { on } 7 \\
9 & T @_{j}(T \leftrightarrow \nabla k) & (\mathrm{F} \square) \text { on } 7 \\
10 & F @_{k}(\mathrm{~T} \rightarrow p) & (\mathrm{F} \square) \text { on } 7 \\
11 & T @_{k}(\square p \rightarrow \perp) & (\mathrm{TE}) \text { on } 2 \\
12 & F @_{k}(\mathrm{~T} \rightarrow \square p) & (\mathrm{T} \leq) \text { on } 11 \\
\vdots & \vdots &
\end{array}\right.
\end{aligned}
$$

Figure 7. Non-termination in the presence of global modalities.

By the basic calculus we will understand the calculus with the rules for the global modalities removed, that is, without (FE), (TE), (TA) and (FA). This gives a calculus for the global modality-free fragment of many-valued hybrid logic. In the following we will prove that the basic calculus is sound, complete and terminating. The termination proof closely resembles the termination proof for the tableau calculus of basic hybrid logic provided in [4]. An important feature of both calculi is that they are terminating even without applying any loop-checks.

If $\alpha$ and $\beta$ are signed formulas on a tableau branch, then $\beta$ is said to be produced by $\alpha$ if $\beta$ is one of the conclusions of a rule application with principal premise $\alpha$. The signed formula $\beta$ is said to be indirectly produced by $\alpha$ if there exists a sequence of signed formulas $\alpha, \alpha_{1}, \alpha_{2}, \ldots, \alpha_{n}, \beta$ in which each formula is produced by its predecessor. We now have the following result.

LEMMA 6 (Quasi-subformula property). Let $\mathcal{T}$ be a tableau in the basic calculus. For any signed formula $\alpha$ occurring on $\mathcal{T}$, one of the following holds:

1. $\alpha$ is a quasi-subformula of the root formula of $\mathcal{T}$.

2. $\alpha$ is a formula of one of the forms $T @_{i}(a \rightarrow \diamond j), T @_{i}(\diamond j \rightarrow a), F @_{i}(a \rightarrow \diamond j)$ or $F @_{i}(\diamond j \rightarrow a)$, for which one of the following holds:

(a) $j$ is a root nominal.

(b) $\alpha$ is indirectly produced by $(\mathrm{F} \square)$ or $(\mathrm{F} \diamond)$ by a number of applications of the reversal rules.

Proof. The proof goes by induction on the construction of $\mathcal{T}$. In the base case, $\alpha$ is just the root formula, which of course is of type 1. For the induction step, assume $\alpha$ has been introduced by applying one of the tableau rules to premises of type 1 or 2 . We need to 
show that then $\alpha$ is also of type 1 or 2 . Consider first the case where $\alpha$ was introduced by applying one of the propositional rules. These rules only take premises of type 1 , and thus also only produce consequences of type 1 . Thus, in this case, $\alpha$ will be of type 1 . Now consider the case where $\alpha$ was produced by applying one of the reversal rules. The reversal rules produce type 1 conclusions from type 1 premises, and type 2 conclusions from type 2 premises. Thus, in this case $\alpha$ will also be of type 1 or 2 . Now assume $\alpha$ was produced by a modal rule. If it was the rule (T $\square)$, then the principal premise of the rule application must have been of type 1 (as the principal premise does not have the form of a type 2 formula). This implies that $\alpha$ is also of type 1 . In case of the rule $(\mathrm{T} \diamond)$, the side condition ensures that the principal premise, and thus the conclusion, is of type 1 . If $\alpha$ is introduced by one of the rules $(\mathrm{F} \square)$ or $(\mathrm{F} \diamond)$, again the premise must be of type 1 . These rules produce two formulas, the first one is by definition of type $2 \mathrm{~b}$ and the second must be of type 1 , since the premise is. Thus in this case $\alpha$ is either of type 1 or type $2 \mathrm{~b}$. Consider finally the case where $\alpha$ was produced by one of the hybrid rules. If it was one of the rules (TRANS), (NOM EQ), $\left(@_{L}\right)$ or $\left(@_{R}\right)$, then the premise of the rule application must have been of type 1 (as the premises do not have the form of a type 2 formula). Thus also $\alpha$ will be of type 1. If it was instead one of the rules (T-NOM) or (F-NOM), then the side condition insures that the principal premise, and thus the conclusion $\alpha$, is of type 1 . If it was either $\left(\mathrm{BRIDGE}_{L}\right)$ or $\left(\mathrm{BRIDGE}_{R}\right)$, then the non-principal premise must have been of type 1 , implying that $j$ is a root nominal. From this we can conclude that the conclusion $\alpha$ must be of type $2 \mathrm{a}$. This completes the proof.

Note that in the basic calculus the only rules that can introduce new nominals to a tableau are $(\mathrm{F} \square)$ and $(\mathrm{F} \diamond)$.

DEFINITION 7. Let $\Theta$ be a branch of a tableau in the basic calculus. If a nominal $j$ has been introduced to the branch by applying either $(\mathrm{F} \square)$ or $(\mathrm{F} \diamond)$ to a premise with prefix $i$, then we say that $j$ is generated by $i$ on $\Theta$, and write $i \prec_{\Theta} j$.

LEMMA 8. Let $\Theta$ be a branch of a tableau in the basic calculus. The graph $G=\left(N^{\Theta}, \prec_{\Theta}\right)$, where $N^{\Theta}$ is the set of nominals occurring on $\Theta$, is a finite set of wellfounded, finitely branching trees.

Proof. That $G$ is wellfounded follows from the observation that if $i \prec_{\Theta} j$, then the first occurrence of $i$ on $\Theta$ is before the first occurrence of $j$. That $G$ is finitely branching is shown as follows. For any given nominal $i$ the number of nominals $j$ satisfying $i \prec \Theta j$ is bounded by the number of applications of $(\mathrm{F} \square)$ and $(\mathrm{F} \diamond)$ to premises of the form $F @_{i}(a \rightarrow \square \varphi)$ and $F @_{i}(\nabla \varphi \rightarrow a)$. So to prove that $G$ is finitely branching, we only need to prove that for any given $i$ the number of such premises is finite. However, this follows immediately from the fact that all such premises must be quasi-subformulas of the root formula (cf. Lemma 6 and the condition on applications of $(\mathrm{F} \diamond)$ ). What is left is to prove that $G$ is a finite set of trees. This follows from the fact that each nominal in $N^{\Theta}$ can be generated by at most one other nominal, and the fact that each nominal in $N^{\Theta}$ must have one of the finitely many root nominals of $\Theta$ as an ancestor.

LEMMA 9. Let $\Theta$ be a branch of a basic tableau. Then $\Theta$ is infinite if and only if there exists an infinite chain of nominals

$$
i_{1} \prec_{\Theta} i_{2} \prec_{\Theta} i_{3} \prec_{\Theta} \cdots .
$$


Proof. The 'if' direction is trivial. To prove the 'only if' direction, let $\Theta$ be any infinite tableau branch. $\Theta$ must contain infinitely many distinct nominals, since it follows immediately from Lemma 6 that a tableau with finitely many nominals can only contain finitely many distinct formulas. This implies that the graph $G=\left(N^{\Theta}, \prec_{\Theta}\right)$ defined as in Lemma 8 must be infinite. Since by Lemma $8, G$ is a finite set of wellfounded, finitely branching trees, $G$ must then contain an infinite path $\left(i_{1}, i_{2}, i_{3}, \ldots\right)$. Thus we get an infinite chain $i_{1} \prec_{\Theta} i_{2} \prec_{\Theta} i_{3} \prec_{\Theta} \cdots$.

DEFINITION 10. Let $\Theta$ be a branch of a tableau in the basic calculus, and let $i$ be a nominal occurring on $\Theta$. We define $m_{\Theta}(i)$ to be the maximal length of any formula with prefix $i$ occurring on $\Theta$.

LEMMA 11 (Decreasing length). Let $\Theta$ be a branch of a basic tableau. If $i \prec_{\Theta} j$ then $m_{\Theta}(i)>m_{\Theta}(j)$.

Proof. For any signed formula $\alpha$, we will use $|\alpha|$ to denote the length of $\alpha$. Assume $i \prec_{\Theta} j$. Let $\alpha$ be a signed formula satisfying: 1) $\alpha$ has maximal length among the formulas on $\Theta$ with prefix $j$;2) $\alpha$ is the earliest occurring formula on $\Theta$ with this property. We need to prove $m_{\Theta}(i)>|\alpha|$. The formula $\alpha$ cannot have been introduced on $\Theta$ by applying any of the propositional rules (Figure 3), since this would contradict maximality of $\alpha$. It cannot have been directly produced by any of the reversal rules (Figure 4) either, since this would contradict the choice of $\alpha$ as the earliest possible on $\Theta$ of maximal length with prefix $j$. By the same argument, $\alpha$ cannot have been directly produced by any of the rules $\left(\mathrm{BRIDGE}_{L}\right),\left(\mathrm{BRIDGE}_{R}\right),($ TRANS) or (NOM EQ). Assume now $\alpha$ has been introduced by applying $\left(@_{L}\right)$ or $\left(@_{R}\right)$ to a premise of the form $T @_{k}\left(@_{j} \varphi \rightarrow a\right)$ or $T @_{k}\left(a \rightarrow @_{j} \varphi\right)$. By Lemma 6 , the premise must be a quasi-subformula of the root formula. Thus $j$ must be a root nominal. However, this is a contradiction, since by assumption $j$ is generated by $i$, and can thus not be a root nominal. Thus neither $\left(@_{L}\right)$ nor $\left(@_{R}\right)$ can have been the rule producing $\alpha$. Now assume that $\alpha$ has been produced by an application of either (F-NOM) or (T-NOM). Since $\alpha$ has index $j$, the non-principal premise used in this rule application must have the form $T @_{i}(a \rightarrow j)$. By Lemma 6 , this premise must be a quasi-subformula of the root formula, and thus $j$ is again a root nominal, which is a contradiction. Thus $\alpha$ can not have been produced by (F-NOM) or (T-NOM) either. Thus $\alpha$ must have been introduced by one of the modal rules $(\mathrm{F} \square),(\mathrm{T} \square),(\mathrm{F} \diamond)$ or $(\mathrm{T} \diamond)$. Consider first the case of the $(\mathrm{F} \square)$ and $(\mathrm{F} \diamond)$ rules. If an instance of one of these produced $\alpha$, then this instance must have been applied to a premise $\beta$ with prefix $i$, since we have assumed $i \prec_{\Theta} j$ and by Lemma 8 there cannot be an $i^{\prime} \neq i$ satisfying $i^{\prime} \prec_{\Theta} j$. (Note that if $\alpha$ is of the form $T @_{j}(b \rightarrow \diamond k)$ or $T @_{j}(\diamond k \rightarrow b)$ produced by a formula $F @_{j}(a \rightarrow \square \varphi)$ or $F @_{j}(\nabla \varphi \rightarrow a)$, this would lead to a contradiction with the assumption that $\alpha$ has maximal length with prefix $j$ and is the earliest occurring formula with this property.) Since the rules in question always produce conclusions that are shorter than their premises, $\beta$ must be longer than $\alpha$. Since $\beta$ is a formula with prefix $i$ we then get:

$$
m_{\Theta}(i) \geq|\beta|>|\alpha|,
$$

as required. Finally, consider the case where $\alpha$ has been produced by either $(\mathrm{T} \square)$ or $(\mathrm{T} \diamond)$. Then $\alpha$ has been produced by a rule instance with non-principal premise of the form $T @_{k}(b \rightarrow \diamond j)$. Since $j$ is not a root nominal, this premise cannot be a quasi-subformula of 
the root formula. Neither can it be of the type 2a mentioned in Lemma 6 . It must thus be of type $2 \mathrm{~b}$, that is, it must be indirectly produced by formulas of the form $T @_{k}\left(b_{m} \rightarrow \diamond j^{\prime}\right)$ or $T @_{k}\left(\diamond j^{\prime} \rightarrow b_{m}\right)$ obtained as conclusion by applications of $(\mathrm{F} \square)$ or $(\mathrm{F} \diamond)$. Since only reversal rules have been applied in the indirect production from these conclusions, we must have $j=j^{\prime}$ and thus $k \prec_{\Theta} j$. Since we already have $i \prec_{\Theta} j$ we get $k=i$, using Lemma 8 . We can conclude that the non-principal premise of the rule instance producing $\alpha$ must have the form $T @_{i}(b \rightarrow \diamond j)$, and thus the principal premise must be a formula $\beta$ with index $i$. Since the rules in question always produce conclusions that are shorter than their premises, $\beta$ must be longer than $\alpha$. Since $\beta$ is a formula with prefix $i$ we then again get the sequence of inequalities (6), as required.

We can now finally prove termination of the basic calculus.

THEOREM 12 (Termination of the basic calculus). Any tableau in the basic calculus is finite.

Proof. Assume there exists an infinite basic tableau. Then it must have an infinite branch $\Theta$. By Lemma 9, there exists an infinite chain

$$
i_{1} \prec_{\Theta} i_{2} \prec_{\Theta} i_{3} \prec_{\Theta} \cdots
$$

Now by Lemma 11 we have

$$
m_{\Theta}\left(i_{1}\right)>m_{\Theta}\left(i_{2}\right)>m_{\Theta}\left(i_{3}\right)>\cdots
$$

which is a contradiction, since $m_{\Theta}(i)$ is a non-negative number for any nominal $i$.

In Figure 7, we provided an example of a non-terminating tableau construction in the calculus including the global modalities. Call the branch of this figure $\Theta$. From the figure it is seen that we have $j \prec_{\Theta} k$ and $m_{\Theta}(j)=m_{\Theta}(k)$. This shows that the part of the termination proof above that would fail in the presence of the global modalities is Lemma 11. Using loop-checks, it would be possible to regain termination even in the presence of the global modalities. This is fairly straightforward, using the method of [4]. However, if we decided to include the global modalities and use loop-checks, the completeness proof would become somewhat more involved.

\section{Completeness of the basic calculus}

In this section we prove completeness of the basic calculus. We will leave the proof of soundness to the reader, as it is fairly straightforward and done in the standard way by checking that each rule preserves satisfiability.

For completeness, let $\Theta$ be an open, saturated branch in the basic calculus (a saturated branch is one to which no further rules apply). We will use this branch to construct a model $\mathcal{M}_{\Theta}=\left\langle W_{\Theta}, R_{\Theta}, \mathbf{n}_{\Theta}, \nu_{\Theta}\right\rangle$. The set of worlds, $W_{\Theta}$ is simply defined to be the set of nominals occurring on $\Theta$. The definition of the other elements of the model requires a bit more work. First, fix a choice function $\sigma$ that to any given set of nominals on $\Theta$ assigns one of these nominals (that is, $\sigma(N) \in N$ for any set $N$ of nominals on $\Theta$ ). We now define the mapping $\mathbf{n}_{\Theta}$ in the following way:

$$
\mathbf{n}_{\Theta}(i)= \begin{cases}\sigma\left\{j \mid T @_{i}(\top \rightarrow j) \in \Theta\right\} & \text { if }\left\{j \mid T @_{i}(T \rightarrow j) \in \Theta\right\} \neq \emptyset \\ i & \text { otherwise. }\end{cases}
$$


LEMMA 13. Let $\Theta$ be a saturated tableau branch. Then we have the following properties:

1. If $T @_{i} \varphi \in \Theta$ is a quasi-subformula of the root formula then $T @_{\mathbf{n}_{\Theta}(i)} \varphi \in \Theta$. Similarly, if $F @_{i} \varphi \in \Theta$ is a quasi-subformula of the root formula then $F @_{\mathbf{n}_{\Theta}(i)} \varphi \in \Theta$.

2. If $T @_{i}(T \rightarrow j) \in \Theta$ then $\mathbf{n}_{\Theta}(i)=\mathbf{n}_{\Theta}(j)$.

Proof. First we prove (i). Assume $T @_{i} \varphi \in \Theta$ is a quasi-subformula of the root formula. If $\mathbf{n}_{\Theta}(i)=i$ then there is nothing to prove. So assume $\mathbf{n}_{\Theta}(i)=\sigma\left\{j \mid T @_{i}(T \rightarrow j) \in \Theta\right\}$. Then $T @_{i}\left(T \rightarrow \mathbf{n}_{\Theta}(i)\right) \in \Theta$, and by applying (T-NOM) to premises $T @_{i} \varphi$ and $T @_{i}(T \rightarrow$ $\left.\mathbf{n}_{\Theta}(i)\right)$ we get $T @_{\mathbf{n}_{\Theta}(i)} \varphi$, as needed. The case of $F @_{i} \varphi \in \Theta$ is proved similarly, using (F-NOM) instead of (T-NOM). We now prove (ii). Assume $T @_{i}(T \rightarrow j) \in \Theta$. To prove $\mathbf{n}_{\Theta}(i)=\mathbf{n}_{\Theta}(j)$ it suffices to prove that for all nominals $k, T @_{i}(T \rightarrow k) \in \Theta \Leftrightarrow T @_{j}(T \rightarrow$ $k) \in \Theta$. So let $k$ be an arbitrary nominal. If $T @_{i}(T \rightarrow k) \in \Theta$ then we can apply (T-NOM) (since $T @_{i}(T \rightarrow k)$ is a quasi-subformula of the root formula by Lemma 6) to premises $T @_{i}(T \rightarrow k)$ and $T @_{i}(T \rightarrow j)$ to obtain the conclusion $T @_{j}(T \rightarrow k)$, as required. If conversely $T @_{j}(T \rightarrow k) \in \Theta$ then we can apply (TRANS) to premises $T @_{i}(T \rightarrow j)$ and $T @_{j}(T \rightarrow k)$ to obtain the conclusion $T @_{i}(T \rightarrow k)$, as required.

We now turn to the definition of $\nu_{\Theta}$. As in [16], we will not define a particular valuation $\nu$ of the propositional variables occuring on the branch, but only show that any valuation assigning values between a certain lower and upper bound (both given by the branch $\Theta$ ) will do. Let us first define these bounds.

DEFINITION 14. For a formula $\varphi$ in the language of MVHL and a nominal $i$, define:

$$
\begin{aligned}
& \text { bound }^{\Theta, i}(\varphi)=\prod\left\{a \mid T @_{i}(\varphi \rightarrow a) \in \Theta\right\} \\
& \text { bound }_{\Theta, i}(\varphi)=\bigsqcup\left\{a \mid T @_{i}(a \rightarrow \varphi) \in \Theta\right\}
\end{aligned}
$$

The intuition is that bound $d^{\Theta, i}(\varphi)$ is an upper bound for the truth-value of $\varphi$ at the world $i$ decided by the branch $\Theta$ and $\operatorname{bound}_{\Theta, i}(\varphi)$ is a lower bound for this truth-value. The following lemma corresponds to Lemma 6.4 of [16] and can be proved in the same way. It ensures that we can actually always choose a value between the lower and the upper bounds.

LEMMA 15. For all $i$ on $\Theta$ and all formulas $\varphi$ of $M V H L$,

$$
\text { bound }_{\Theta, i}(\varphi) \leq \text { bound }^{\Theta, i}(\varphi) .
$$

Later we will show that any valuation assigning a value to $p$ between $\operatorname{bound}_{\Theta, \mathbf{n}_{\Theta}(i)}(p)$ and bound $d^{\Theta, \mathbf{n}_{\Theta}(i)}(p)$ at the world $\mathbf{n}_{\Theta}(i)$ will do for the truth-value of $p$ at this world. The following lemma corresponds to Proposition 6.5 in [16] and is proven in the same way.

LEMMA 16. Let $\varphi$ be any formula in MVHL over $\mathcal{H}$ other than a propositional constant from $\mathcal{H}$, and let $a \in \mathcal{H}$. Then the following holds:

1. If $T @_{i}(a \rightarrow \varphi) \in \Theta$, then $a \leq$ bound $_{\Theta, i}(\varphi)$. 
2. If $T @_{i}(\varphi \rightarrow a) \in \Theta$, then bound ${ }^{\Theta, i}(\varphi) \leq a$.

3. If $F @_{i}(a \rightarrow \varphi) \in \Theta$, then $a \not \leq$ bound $^{\Theta, i}(\varphi)$.

4. If $F @_{i}(\varphi \rightarrow a) \in \Theta$, then bound,$i(\varphi) \not \leq a$.

The accessibility relation $R_{\Theta}$ is defined as follows:

$$
R_{\Theta}(i, j)=\bigsqcup\left\{b \mid T @_{i}(b \rightarrow \diamond k) \in \Theta, \mathbf{n}_{\Theta}(k)=j\right\} .
$$

We have the following result, which we are going to use when proving completeness.

LEMMA 17. If $T @_{i}(c \leftrightarrow \diamond j) \in \Theta$ then $R_{\Theta}\left(i, \mathbf{n}_{\Theta}(j)\right)=c$.

Proof. We will prove $R_{\Theta}\left(i, \mathbf{n}_{\Theta}(j)\right) \geq c$ and $R_{\Theta}\left(i, \mathbf{n}_{\Theta}(j)\right) \leq c$. First we prove $R_{\Theta}\left(i, \mathbf{n}_{\Theta}(j)\right) \geq$ $c$. Since $T @_{i}(c \leftrightarrow \diamond j) \in \Theta$ we have $T @_{i}(c \rightarrow \diamond j) \in \Theta$, and thus

$$
\begin{aligned}
R_{\Theta}\left(i, \mathbf{n}_{\Theta}(j)\right) & =\bigsqcup\left\{b \mid T @_{i}(b \rightarrow \diamond k) \in \Theta, \mathbf{n}_{\Theta}(k)=\mathbf{n}_{\Theta}(j)\right\} \\
& \geq \bigsqcup\left\{b \mid T @_{i}(b \rightarrow \diamond j) \in \Theta\right\} \\
& \geq c .
\end{aligned}
$$

We now prove $R_{\Theta}\left(i, \mathbf{n}_{\Theta}(j)\right) \leq c$. By definition of $\mathbf{n}_{\Theta}$ we have either $\mathbf{n}_{\Theta}(j)=j$ or $T @_{j}(T \rightarrow$ $\left.\mathbf{n}_{\Theta}(j)\right) \in \Theta$. If $T @_{j}\left(T \rightarrow \mathbf{n}_{\Theta}(j)\right) \in \Theta$ then since $T @_{i}(\diamond j \rightarrow c) \in \Theta$ we get $T @_{i}\left(\diamond \mathbf{n}_{\Theta}(j) \rightarrow\right.$ $c) \in \Theta$, using $\left(\right.$ BRIDGE $\left._{L}\right)$. If $\mathbf{n}_{\Theta}(j)=j$ we obviously also have $T @_{i}\left(\diamond \mathbf{n}_{\Theta}(j) \rightarrow c\right) \in \Theta$. Applying item 2 of Lemma 16 we then get bound ${ }^{\Theta, i}\left(\diamond \mathbf{n}_{\Theta}(j)\right) \leq c$. Thus

$$
\begin{aligned}
R_{\Theta}\left(i, \mathbf{n}_{\Theta}(j)\right) & =\bigsqcup\left\{b \mid T @_{i}(b \rightarrow \diamond k) \in \Theta, \mathbf{n}_{\Theta}(k)=\mathbf{n}_{\Theta}(j)\right\} \\
& \left.\leq \bigsqcup\left\{b \mid T @_{i}\left(b \rightarrow \diamond \mathbf{n}_{\Theta}(j)\right) \in \Theta\right\} \quad \text { (using }\left(\operatorname{BRIDGE}_{R}\right)\right) \\
& =\text { bound }_{\Theta, i}\left(\diamond \mathbf{n}_{\Theta}(j)\right) \\
& \leq \text { bound }^{\Theta}\left(\diamond \mathbf{n}_{\Theta}(j)\right) \quad \text { (using Lemma 15) } \\
& \leq c,
\end{aligned}
$$

as required.

The theorem we need for completeness may now be stated in the following way:

THEOREM 18. Let $\nu$ be a valuation such that for all propositional variables $p$ and all nominals $i$ :

$$
\text { bound }_{\Theta, \mathbf{n}_{\Theta}(i)}(p) \leq \nu\left(\mathbf{n}_{\Theta}(i), p\right) \leq \text { bound }^{\Theta, \mathbf{n}_{\Theta}(i)}(p) .
$$

Then for all quasi-subformulas $\varphi$ of the root formula:

$$
\text { bound }_{\Theta, \mathbf{n}_{\Theta}(i)}(\varphi) \leq \nu\left(\mathbf{n}_{\Theta}(i), \varphi\right) \leq \text { bound }^{\Theta, \mathbf{n}_{\Theta}(i)}(\varphi) .
$$


Proof. By induction on $\varphi$. The base cases are where $\varphi$ is a propositional variable $p$, a value $c \in \mathcal{H}$ or a nominal $j$. The case where $\varphi$ is $p$ follows directly by the assumption. The case where $\varphi$ is $c$ is easy: First note that for any truth-values $a, b$, if $T @_{\mathbf{n}_{\Theta}(i)}(a \rightarrow b) \in \Theta$ then $a \leq b$. This follows from closure rule 1 presented in Section 4 . Thus we get:

$$
\begin{aligned}
\text { bound }_{\Theta, \mathbf{n}_{\Theta}(i)}(c) & =\bigsqcup\left\{a \mid T @_{\mathbf{n}_{\Theta}(i)}(a \rightarrow c) \in \Theta\right\} \leq c \leq \prod\left\{a \mid T @_{\mathbf{n}_{\Theta}(i)}(c \rightarrow a) \in \Theta\right\} \\
& =\text { bound }^{\Theta, \mathbf{n}_{\Theta}(i)}(c) .
\end{aligned}
$$

Now assume $\varphi$ is a nominal $j$. By definition of $\nu, \nu\left(\mathbf{n}_{\Theta}(i), j\right)$ is $\top$ if $\mathbf{n}_{\Theta}(j)=\mathbf{n}_{\Theta}(i)$ and $\perp$ otherwise. Assume first $\mathbf{n}_{\Theta}(j)=\mathbf{n}_{\Theta}(i)$. Then $\nu\left(\mathbf{n}_{\Theta}(i), j\right)$ is $\top$, so trivially we have bound $_{\Theta, \mathbf{n}_{\Theta}(i)}(j) \leq \nu\left(\mathbf{n}_{\Theta}(i), j\right)$. We thus only need to prove $\nu\left(\mathbf{n}_{\Theta}(i), j\right) \leq$ bound $^{\Theta, \mathbf{n}_{\Theta}(i)}(j)$, that is, we need to prove $\top=$ bound $^{\Theta, \mathbf{n}_{\Theta}(i)}(j)=\prod\left\{a \mid T @_{\mathbf{n}_{\Theta}(i)}(j \rightarrow a) \in \Theta\right\}$. This amounts to show that, for all $a \in \mathcal{H}, T @_{\mathbf{n}_{\Theta}(i)}(j \rightarrow a) \in \Theta$ implies $a=\top$. Assume towards a contradiction that, for some $a, T @_{\mathbf{n}_{\Theta}(i)}(j \rightarrow a) \in \Theta$ and $a \neq \top$. Since we have assumed $\mathbf{n}_{\Theta}(j)=\mathbf{n}_{\Theta}(i)$, by definition of $\mathbf{n}_{\Theta}$ we get that either $j=\mathbf{n}_{\Theta}(i)$ or $T @_{j}\left(T \rightarrow \mathbf{n}_{\Theta}(i)\right) \in \Theta$. If $j=\mathbf{n}_{\Theta}(i)$ then we have that $\Theta$ contains a formula of the form $T @_{\mathbf{n}_{\Theta}(i)}\left(\mathbf{n}_{\Theta}(i) \rightarrow a\right)$ where $a \neq T$. This immediately contradicts closure rule 7. Assume instead $T @_{j}(T \rightarrow$ $\left.\mathbf{n}_{\Theta}(i)\right) \in \Theta$. Since we also have $T @_{\mathbf{n}_{\Theta}(i)}(j \rightarrow a) \in \Theta$ where $a \neq \top$, we can apply $(\mathrm{T} \leq)$ to conclude that that $\Theta$ must contain a formula of the form $F @_{\mathbf{n}_{\Theta}(i)}(t \rightarrow j)$ where $t$ is some truth-value different from $\perp$. Since $\Theta$ then contains both $T @_{j}\left(T \rightarrow \mathbf{n}_{\Theta}(i)\right)$ and $F @_{\mathbf{n}_{\Theta}(i)}(t \rightarrow j)$ where $t \neq \perp$, we get a contradiction by closure rule 6 . Assume now $\mathbf{n}_{\Theta}(j) \neq \mathbf{n}_{\Theta}(i)$. Then $\nu\left(\mathbf{n}_{\Theta}(i), j\right)=\perp$, and the inequality $\nu\left(\mathbf{n}_{\Theta}(i), j\right) \leq$ bound $^{\Theta, \mathbf{n}_{\Theta}(i)}(j)$ thus holds trivially. To prove the other inequality, bound $\cos _{\Theta}(i)(j) \leq \nu\left(\mathbf{n}_{\Theta}(i), j\right)$, we need to show that if $T @_{\mathbf{n}_{\Theta}(i)}(a \rightarrow j) \in \Theta$ then $a=\perp$. Thus assume toward a contradiction that $T @_{\mathbf{n}_{\Theta}(i)}(a \rightarrow j) \in \Theta$ and $a \neq \perp$. Then rule (NOM EQ) implies $T @_{\mathbf{n}_{\Theta}(i)}(T \rightarrow j) \in \Theta$. Thus, by item 2 of Lemma 13, we get $\mathbf{n}_{\Theta}(i)=\mathbf{n}_{\Theta}(j)$, contradicting the assumption.

Now for the induction step. The propositional cases, that is, where $\varphi$ is a conjunction, disjunction or implication, are all simple and thus omitted. Now consider the case where $\varphi$ is $@_{j} \psi$. Note that $\nu\left(\mathbf{n}_{\Theta}(i), @_{j} \psi\right)=\nu\left(\mathbf{n}_{\Theta}(j), \psi\right)$ and by induction hypothesis,

$$
\text { bound }_{\Theta, \mathbf{n}_{\Theta}(j)}(\psi) \leq \nu\left(\mathbf{n}_{\Theta}(j), \psi\right) \leq \text { bound }^{\Theta, \mathbf{n}_{\Theta}(j)}(\psi) .
$$

Now by the rule $\left(@_{R}\right)$, if $T @_{\mathbf{n}_{\Theta}(i)}\left(a \rightarrow @_{j} \psi\right) \in \Theta$ then $T @_{j}(a \rightarrow \psi) \in \Theta$, for all $a \in \mathcal{H}$. Thus we get that

$$
\begin{aligned}
\text { bound }_{\Theta, \mathbf{n}_{\Theta}(i)}\left(@_{j} \psi\right) & =\bigsqcup\left\{a \mid T @_{\mathbf{n}_{\Theta}(i)}\left(a \rightarrow @_{j} \psi\right) \in \Theta\right\} \\
& \leq \bigsqcup\left\{a \mid T @_{j}(a \rightarrow \psi) \in \Theta\right\} \\
& \leq \bigsqcup\left\{a \mid T @_{\mathbf{n}_{\Theta}(j)}(a \rightarrow \psi) \in \Theta\right\} \quad \text { (using 1 of Lemma 13) } \\
& =\operatorname{bound}_{\Theta, \mathbf{n}_{\Theta}(j)}(\psi) \\
& \leq \nu\left(\mathbf{n}_{\Theta}(j), \psi\right) \\
& =\nu\left(\mathbf{n}_{\Theta}(i), @_{j} \psi\right) .
\end{aligned}
$$

By a completely symmetric argument, using the rule $\left(@_{L}\right)$ instead of $\left(@_{R}\right)$, it can shown that

$$
\nu\left(\mathbf{n}_{\Theta}(i), @_{j} \psi\right) \leq \text { bound }^{\Theta, \mathbf{n}_{\Theta}(i)}\left(@_{j} \psi\right) .
$$


This completes the @-case.

In case $\varphi$ is $\diamond \psi$, we need to prove that

$$
\text { bound }_{\Theta, \mathbf{n}_{\Theta}(i)}(\diamond \psi) \leq \nu\left(\mathbf{n}_{\Theta}(i), \diamond \psi\right) \leq \text { bound }^{\Theta, \mathbf{n}_{\Theta}(i)}(\diamond \psi),
$$

which by definition amounts to showing that

$$
\begin{aligned}
\bigsqcup\left\{a \mid T @_{\mathbf{n}_{\Theta}(i)}(a \rightarrow \diamond \psi) \in \Theta\right\} & \leq \bigsqcup\left\{R_{\Theta}\left(\mathbf{n}_{\Theta}(i), j\right) \sqcap \nu(j, \psi) \mid j \in \Theta\right\} \\
& \leq \prod\left\{a \mid T @_{\mathbf{n}_{\Theta}(i)}(\diamond \psi \rightarrow a) \in \Theta\right\} .
\end{aligned}
$$

Proving the first inequality amounts to showing that if $T @_{\mathbf{n}_{\Theta}(i)}(a \rightarrow \diamond \psi) \in \Theta$ then

$$
a \leq \bigsqcup\left\{R_{\Theta}\left(\mathbf{n}_{\Theta}(i), j\right) \sqcap \nu(j, \psi) \mid j \in \Theta\right\} .
$$

Assume towards a contradiction that

$$
T @_{\mathbf{n}_{\Theta}(i)}(a \rightarrow \diamond \psi) \in \Theta \text { and } a \not \leq \bigsqcup\left\{R_{\Theta}\left(\mathbf{n}_{\Theta}(i), j\right) \sqcap \nu(j, \psi) \mid j \in \Theta\right\},
$$

for some $a \in \mathcal{H}$. Then choose a $b \in \mathcal{H}$ such that $b \geq \bigsqcup\left\{R_{\Theta}\left(\mathbf{n}_{\Theta}(i), j\right) \sqcap \nu(j, \psi) \mid j \in\right.$ $\Theta\}$ and $b$ is a maximal member of $\mathcal{H}$ with $a \not \leq b$. Then by the reversal rule $(\mathrm{T} \geq)$, $F @_{\mathbf{n}_{\Theta}(i)}(\diamond \psi \rightarrow b) \in \Theta$. Then using the $(\mathrm{F} \diamond)$ rule there is a $c \in \mathcal{H}$ and a $k \in \Theta$ such that $T @_{\mathbf{n}_{\Theta}(i)}(c \leftrightarrow \diamond k) \in \Theta$ and $F @_{k}(\varphi \rightarrow(c \Rightarrow b)) \in \Theta$. Since $T @_{\mathbf{n}_{\Theta}(i)}(c \leftrightarrow \Delta k) \in$ $\Theta$, Lemma 17 implies $R_{\Theta}\left(\mathbf{n}_{\Theta}(i), \mathbf{n}_{\Theta}(k)\right)=c$. Applying 1 of Lemma 13 to the formula $F @_{k}(\varphi \rightarrow(c \Rightarrow b)) \in \Theta$ we get $F @_{\mathbf{n}_{\Theta}(k)}(\varphi \rightarrow(c \Rightarrow b)) \in \Theta$. Now item 4 of Lemma 16

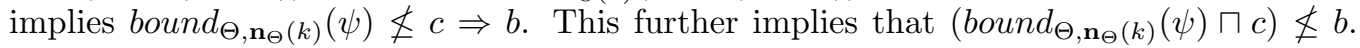
But by the induction hypothesis bound $_{\Theta, \mathbf{n}_{\Theta}(k)}(\psi) \leq \nu\left(\mathbf{n}_{\Theta}(k), \psi\right)$ and thus

$$
\begin{aligned}
\text { bound }_{\Theta, \mathbf{n}_{\Theta}(k)}(\psi) \sqcap c & =\operatorname{bound}_{\Theta, \mathbf{n}_{\Theta}(k)}(\psi) \sqcap R_{\Theta}\left(\mathbf{n}_{\Theta}(i), \mathbf{n}_{\Theta}(k)\right) \\
& \leq \nu\left(\mathbf{n}_{\Theta}(k), \psi\right) \sqcap R_{\Theta}\left(\mathbf{n}_{\Theta}(i), \mathbf{n}_{\Theta}(k)\right) \\
& \leq \bigsqcup\left\{R_{\Theta}\left(\mathbf{n}_{\Theta}(i), \mathbf{n}_{\Theta}(j)\right) \sqcap \nu\left(\mathbf{n}_{\Theta}(j), \psi\right) \mid j \in \Theta\right\} \\
& \leq \bigsqcup\left\{R_{\Theta}\left(\mathbf{n}_{\Theta}(i), j\right) \sqcap \nu(j, \psi) \mid j \in \Theta\right\} \leq b,
\end{aligned}
$$

which of course is a contradiction.

In order to prove that

$$
\bigsqcup\left\{R_{\Theta}\left(\mathbf{n}_{\Theta}(i), j\right) \sqcap \nu(j, \psi) \mid j \in \Theta\right\} \leq \prod\left\{a \mid T @_{\mathbf{n}_{\Theta}(i)}(\diamond \psi \rightarrow a) \in \Theta\right\},
$$

we must show that if $T @_{\mathbf{n}_{\Theta}(i)}(\diamond \psi \rightarrow a) \in \Theta$, then $R_{\Theta}\left(\mathbf{n}_{\Theta}(i), j\right) \sqcap \nu(j, \psi) \leq a$ for all $j \in \Theta$. Thus assume that $T @_{\mathbf{n}_{\Theta}(i)}(\diamond \psi \rightarrow a) \in \Theta$ and that $R_{\Theta}\left(\mathbf{n}_{\Theta}(i), j\right) \neq \perp$ (or else it's trivial) for an arbitrary $j \in \Theta$. By definition,

$$
R_{\Theta}\left(\mathbf{n}_{\Theta}(i), j\right)=\bigsqcup\left\{b \mid T @_{\mathbf{n}_{\Theta}(i)}(b \rightarrow \diamond k) \in \Theta, \mathbf{n}_{\Theta}(k)=j\right\} .
$$

Since $R\left(\mathbf{n}_{\Theta}(i), j\right) \neq \perp$, there exists $b$ and $k$ such that $T @_{\mathbf{n}_{\Theta}(i)}(b \rightarrow \diamond k) \in \Theta$ and $\mathbf{n}_{\Theta}(k)=j$. Let such $b$ and $k$ be chosen arbitrarily. Then by the $(\mathrm{T} \diamond)$ rule, $T @_{k}(\psi \rightarrow(b \Rightarrow a)) \in \Theta$. Using 1 of Lemma 13 we get $T @_{\mathbf{n}_{\Theta}(k)}(\psi \rightarrow(b \Rightarrow a)) \in \Theta$ Now, by induction hypothesis,

$$
\nu(j, \psi)=\nu\left(\mathbf{n}_{\Theta}(k), \psi\right) \leq \text { bound }^{\Theta, \mathbf{n}_{\Theta}(k)}(\psi) \leq b \Rightarrow a .
$$


Since $k$ and $b$ were chosen arbitrarily with $T @_{\mathbf{n}_{\Theta}(i)}(b \rightarrow \diamond k) \in \Theta$ and $\mathbf{n}_{\Theta}(k)=j$, we get

$$
\nu(j, \psi) \leq \prod\left\{b \Rightarrow a \mid T @_{\mathbf{n}_{\Theta}(i)}(b \rightarrow \diamond k) \in \Theta, \mathbf{n}_{\Theta}(k)=j\right\} .
$$

We now get

$$
\begin{aligned}
R_{\Theta}\left(\mathbf{n}_{\Theta}(i), j\right) \sqcap \nu(j, \psi) \leq & \bigsqcup\left\{b \mid T @_{\mathbf{n}_{\Theta}(i)}(b \rightarrow \diamond k) \in \Theta, \mathbf{n}_{\Theta}(k)=j\right\} \\
& \sqcap \prod\left\{b \Rightarrow a \mid T @_{\mathbf{n}_{\Theta}(i)}(b \rightarrow \diamond k) \in \Theta, \mathbf{n}_{\Theta}(k)=j\right\} \\
\leq & \bigsqcup\left\{b \sqcap(b \Rightarrow a) \mid T @_{\mathbf{n}_{\Theta}(i)}(b \rightarrow \diamond k) \in \Theta, \mathbf{n}_{\Theta}(k)=j\right\} \\
\leq & \bigsqcup\left\{a \mid T @_{\mathbf{n}_{\Theta}(i)}(b \rightarrow \diamond k) \in \Theta, \mathbf{n}_{\Theta}(k)=j\right\} \\
\leq & a .
\end{aligned}
$$

Because $j \in \Theta$ was arbitrary it follows that it holds for all $j \in \Theta$ and the proof of this case is completed.

In case $\varphi$ is $\square \psi$, we need to prove that

$$
\begin{aligned}
\bigsqcup\left\{a \mid T @_{\mathbf{n}_{\Theta}(i)}(a \rightarrow \square \psi) \in \Theta\right\} & \leq \prod\left\{R_{\Theta}\left(\mathbf{n}_{\Theta}(i), j\right) \Rightarrow \nu(j, \psi) \mid j \in \Theta\right\} \\
& \leq \prod\left\{a \mid T @_{\mathbf{n}_{\Theta}(i)}(\square \psi \rightarrow a) \in \Theta\right\} .
\end{aligned}
$$

To prove the first inequality we need to prove that if $j \in \Theta$, then

$$
a \leq R_{\Theta}\left(\mathbf{n}_{\Theta}(i), j\right) \Rightarrow \nu(j, \psi),
$$

for all $a \in \mathcal{H}$ with $T @_{\mathbf{n}_{\Theta}(i)}(a \rightarrow \square \psi) \in \Theta$. So let $a \in \mathcal{H}$ be given arbitrarily such that $T @_{\mathbf{n}_{\Theta}(i)}(a \rightarrow \square \psi) \in \Theta$. Note that (7) is equivalent to

$$
a \sqcap R_{\Theta}\left(\mathbf{n}_{\Theta}(i), j\right) \leq \nu(j, \psi) .
$$

By definition of $R_{\Theta}$ we have

$$
R_{\Theta}\left(\mathbf{n}_{\Theta}(i), j\right)=\bigsqcup\left\{b \mid T @_{\mathbf{n}_{\Theta}(i)}(b \rightarrow \diamond k) \in \Theta, \mathbf{n}_{\Theta}(k)=j\right\} .
$$

Let $b$ and $k$ be chosen arbitrarily such that $T @_{\mathbf{n}_{\Theta}(i)}(b \rightarrow \diamond k) \in \Theta$ and $\mathbf{n}_{\Theta}(k)=j$. Then by the (T $\square$ )-rule it follows that $T @_{k}((a \sqcap b) \rightarrow \psi) \in \Theta$. By 1 of Lemma 13 this implies $T @_{\mathbf{n}_{\Theta}(k)}((a \sqcap b) \rightarrow \psi) \in \Theta$. Thus we get bound $d_{\Theta, \mathbf{n}_{\Theta}(k)}(\psi) \geq(a \sqcap b)$. Since $\mathbf{n}_{\Theta}(k)=j$ we then get, using the induction hypothesis,

$$
\nu(j, \psi)=\nu\left(\mathbf{n}_{\Theta}(k), \psi\right) \geq \text { bound }_{\Theta, \mathbf{n}_{\Theta}(k)}(\psi) \geq(a \sqcap b) .
$$

Since $b$ and $k$ were chosen arbitrarily with the properties $T @_{\mathbf{n}_{\Theta}(i)}(b \rightarrow \diamond k) \in \Theta$ and $\mathbf{n}_{\Theta}(k)=j$ this implies

$$
\begin{aligned}
\nu(j, \psi) & \geq \bigsqcup\left\{a \sqcap b \mid T @_{\mathbf{n}_{\Theta}(i)}(b \rightarrow \diamond k) \in \Theta, \mathbf{n}_{\Theta}(k)=j\right\} \\
& =a \sqcap \bigsqcup\left\{b \mid T @_{\mathbf{n}_{\Theta}(i)}(b \rightarrow \diamond k) \in \Theta, \mathbf{n}_{\Theta}(k)=j\right\} \\
& =a \sqcap R_{\Theta}\left(\mathbf{n}_{\Theta}(i), j\right) .
\end{aligned}
$$


Since $a$ was arbitrary this holds for all $a \in \mathcal{H}$ and the inequality have been proven.

To show the other inequality we need to show that

$$
\text { if } T @_{\mathbf{n}_{\Theta}(i)}(\square \psi \rightarrow a) \in \Theta \text { then } \prod\left\{R_{\Theta}\left(\mathbf{n}_{\Theta}(i), j\right) \Rightarrow \nu(j, \psi) \mid j \in \Theta\right\} \leq a .
$$

If $a=\top$ then this is trivial. Thus assume towards a contradiction that there is an $a \neq \top$ with $T @_{\mathbf{n}_{\Theta}(i)}(\square \psi \rightarrow a) \in \Theta$ and $\prod\left\{R_{\Theta}\left(\mathbf{n}_{\Theta}(i), j\right) \Rightarrow \nu(j, \psi) \mid j \in \Theta\right\} \not \leq a$. Now let $b \leq \prod\left\{R_{\Theta}\left(\mathbf{n}_{\Theta}(i), j\right) \Rightarrow \nu(j, \psi) \mid j \in \Theta\right\}$ be a minimal member of $\mathcal{H}$ such that $b \not \leq a$.

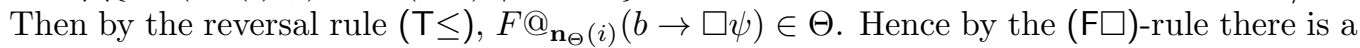
nominal $k \in \Theta$ and a $c \in \mathcal{H}$ such that $T @_{\mathbf{n}_{\Theta}(i)}(c \leftrightarrow \diamond k) \in \Theta$ and $F @_{k}((b \sqcap c) \rightarrow \psi) \in \Theta$. From the first it follows that $R_{\Theta}\left(\mathbf{n}_{\Theta}(i), \mathbf{n}_{\Theta}(k)\right)=c$, using Lemma 17. From the second it follows that $F @_{\mathbf{n}_{\Theta}(k)}((b \sqcap c) \rightarrow \psi) \in \Theta$, using 1 of Lemma 13, and thus, by item 3 of

Lemma 16, $b \sqcap c \not \leq$ bound $^{\Theta, \mathbf{n}_{\Theta}(k)}(\psi)$. But then from the induction hypothesis it follows that

$$
b \sqcap c \not \nu\left(\mathbf{n}_{\Theta}(k), \psi\right) \leq \text { bound }^{\Theta, \mathbf{n}_{\Theta}(k)}(\psi) .
$$

Hence

$$
b \not \leq c \Rightarrow \nu\left(\mathbf{n}_{\Theta}(k), \psi\right)=R_{\Theta}\left(\mathbf{n}_{\Theta}(i), \mathbf{n}_{\Theta}(k)\right) \Rightarrow \nu\left(\mathbf{n}_{\Theta}(k), \psi\right) .
$$

But by the assumption on $b$ we also have that

$$
b \leq \prod\left\{R_{\Theta}\left(\mathbf{n}_{\Theta}(i), j\right) \Rightarrow \nu(j, \psi) \mid j \in \Theta\right\} \leq R_{\Theta}\left(\mathbf{n}_{\Theta}(i), \mathbf{n}_{\Theta}(k)\right) \Rightarrow \nu\left(\mathbf{n}_{\Theta}(k), \psi\right),
$$

and a contradiction have been reached. This concludes the $\square$-case and thus the entire proof of the theorem.

Now completeness can easily be proven, in the following sense.

THEOREM 19. If there is no tableau proof of the formula $\varphi$, then there is a model $\mathcal{M}=$ $\langle W, R, \mathbf{n}, \nu\rangle$ and $a w \in W$ such that $\nu(w, \varphi) \neq \top$.

Proof. Assume that there is no tableau proof of the formula $\varphi$. Then there is a saturated tableau with an open branch $\Theta$ starting with the formula $F @_{i}(T \rightarrow \varphi)$ for a nominal $i$ not in $\varphi$. By item 1 of Lemma 13 it follows that also $F @_{\mathbf{n}_{\Theta}(i)}(T \rightarrow \varphi) \in \Theta$.

The model $\mathcal{M}_{\Theta}=\left\langle W_{\Theta}, R_{\Theta}, \mathbf{n}_{\Theta}, \nu_{\Theta}\right\rangle$ can now be constructed such that $\nu_{\Theta}$ satisfies the assumption of Theorem 18. Since $F @_{\mathbf{n}_{\Theta}(i)}(T \rightarrow \varphi) \in \Theta$ it follows by item 3 Lemma 16 that $T \not \leq$ bound $d^{\Theta, \mathbf{n}_{\Theta}(i)}(\varphi)$. But by Theorem 18, since $\varphi$ is a subformula of the body of the root formula, we know that $\nu_{\Theta}\left(\mathbf{n}_{\Theta}(i), \varphi\right) \leq$ bound $^{\Theta, \mathbf{n}_{\Theta}(i)}(\varphi)$, and it thus follows that $\top \not \leq \nu_{\Theta}\left(\mathbf{n}_{\Theta}(i), \varphi\right)$ and the proof is completed.

\section{Conclusion and future work}

We have presented a many-valued semantics for hybrid logic, being a hybrid version of a many-valued semantics for modal logic provided in $[14,15,16]$. We discussed alternative definitions of the semantics for nominals and satisfaction operators in Section 2.3, and concluded that the chosen semantics is the one that resemble classical hybrid logic most. The exact relationship between our chosen logic and the five alternative logics of Section 
2.3 , and whether they are truly different logics (i.e. whether they constitute different sets of validities), is left to future work. To answer this question a notion of bisimulation would be useful. In fact, bisimulation for the modal logic part of MVHL is discussed in the paper [11] and could thus serve as a starting point for future research.

We presented a sound, complete, and terminating tableau system for the basic logic without the global modalities, which gives rise to a decision procedure for the logic. Furthermore, since our tableau calculus is terminating and provides finite counter models, it also shows that the logic has the finite model property, by which we mean that for every formula which is not valid, there is a finite model in which the formula does not have the truth-value $T$ in some world.

As mentioned in section 5, termination of the tableau system including the rules for the global modalities can be obtained by using loop-checks, however this complicates the completeness proof. Without loop-checks, the completeness proof is a simple extension of the proof in section 6 .

\section{Acknowledgment}

This work is partially funded by the Danish Natural Science Research Council (the HYLOCORE project). The paper is a significantly revised and updated version of [25]. We wish to thank the anonymous reviewers for many suggestions for improvements.

\section{BIBLIOGRAPHY}

[1] P. Blackburn and M. Marx. Tableaux for quantified hybrid logic. In U. Egly and C. Fermüller, editors, Automated Reasoning with Analytic Tableaux and Related Methods, TABLEAUX 2002, volume 2381 of Lecture Notes in Artificial Intelligence, pages 38-52. Springer-Verlag, 2002.

[2] Patrick Blackburn. Internalizing labelled deduction. Journal of Logic and Computation, 10(1):137-168, 2000.

[3] Thomas Bolander and Patrick Blackburn. Terminating tableau calculi for hybrid logics extending K. In Carlos Areces and Stéphane Demri, editors, Workshop Proceedings of Methods for Modalities 5, pages 157-175. École Normale Supérieure de Cachan, France, 2007.

[4] Thomas Bolander and Patrick Blackburn. Termination for hybrid tableaus. Journal of Logic and Computation, 17(3):517-554, 2007.

[5] Thomas Bolander and Torben Braüner. Tableau-based decision procedures for hybrid logic. Journal of Logic and Computation, 16(6):737-763, 2006.

[6] Torben Braüner. Why does the proof-theory of hybrid logic work so well? Journal of Applied NonClassical Logics, 17(4):521-543, 2007.

[7] Torben Braüner. Hybrid Logic and Its Proof-Theory. Applied Logic Series, Vol. 37. Springer, 2011.

[8] Torben Braüner and Valeria de Paiva. Towards constructive hybrid logic (extended abstract). In Carlos Areces and Patrick Blackburn, editors, Workshop Proceedings of Methods for Modalities 3. 2003.

[9] Torben Braüner and Valeria de Paiva. Intuitionistic hybrid logic. Journal of Applied Logic, 4:231-255, 2006. Revised and extended version of [8].

[10] Rohit Chadha, Damiano Macedonio, and Vladimiro Sassone. A hybrid intuitionistic logic: Semantics and decidability. Journal of Logic and Computation, 16(1):27-59, 2006.

[11] Pantelis E. Eleftheriou, Costas D. Koutras, and Christos Nomikos. Notions of bisimulation for heyting-valued modal languages. Journal of Logic and Computation, 2010. Published online, doi:10.1093/logcom/exq005.

[12] W. B. Ewald. Intuitionistic tense and modal logic. Journal of Symbolic Logic, 51:166-179, 1986.

[13] Melvin Fitting. Proof methods for modal and intuitionistic logics. Synthese Library volume 169. D. Reidel Publishing Co., Dordrecht, 1983.

[14] Melvin Fitting. Many-valued modal logics. Fundamenta Informaticae, 15:235-254, 1992.

[15] Melvin Fitting. Many-valued modal logics II. Fundamenta Informaticae, 17:55-73, 1992.

[16] Melvin Fitting. Tableaus for many-valued modal logic. Studia Logica, 55:63-87, 1995. 
[17] Melvin Fitting. Modal proof theory. In Patrick Blackburn, Johan van Benthem, and Frank Wolter, editors, Handbook of Modal Logic, pages 85-138. Elsevier, Amsterdam, 2007.

[18] Melvin Fitting. How true it is = who says it's true. Studia Logica, 91(3):335-266, 2009.

[19] Didier Galmiche and Yakoub Salhi. A family of Gödel hybrid logics. Journal of Applied Logic, 8:371385, 2010.

[20] Didier Galmiche and Yakoub Salhi. Sequent calculi and decidability for intuitionistic hybrid logic. Information and Computation, 209(12):1447-1463, 2011.

[21] Lluís Godo and Ricardo O Rodríguez. Logical approaches to fuzzy similarity-based reasoning: an overview. In Preferences and Similarities, pages 75-128. Springer, 2008.

[22] Jens Ulrik Hansen. A tableau system for a first-order hybrid logic. In Proceedings of the International Workshop on Hybrid Logic (HyLo 2007), pages 32-40. ESSLLI 2007, 2007.

[23] Jens Ulrik Hansen. A hybrid public announcement logic with distributed knowledge. Electronic Notes in Theoretical Computer Science, 273:33-50, 2011. Post-Proceedings of the International Workshop on Hybrid Logic and Applications (HyLo 2010).

[24] Jens Ulrik Hansen. A logic toolbox for modeling knowledge and information in multi-agent systems and social epistemology. PhD thesis, Roskide University, Roskilde, Denmark, 2011. Roskilde University Computer Science Research Report no. 133.

[25] Jens Ulrik Hansen, Thomas Bolander, and Torben Braüner. Many-valued hybrid logic. In Carlos Areces and Robert Goldblatt, editors, Advances in Modal Logic, Volume 7, pages 111-132. College Publications, 2008.

[26] Yoshihiro Maruyama. Reasoning about fuzzy belief and common belief: With emphasis on incomparable beliefs. In Toby Walsh, editor, Proceedings of the Twenty-Second International Joint Conference on Artificial Intelligence (IJCAI-11). AAAI Press/International Joint Conferences on Artificial Intelligence, 2011.

[27] Hiroakira Ono and Nobu-Yuki Suzuki. Relations between intuitionistic modal logics and intermediate predicate logics. Reports on Mathematical Logic, 22:65-87, 1988.

[28] Anne Sjerp Troelstra and Dirk Van Dalen. Constructivism in mathematics, volume 1. Elsevier Science, 1988. 